\title{
High-Frequency EMI Attenuation at Source with the Auxiliary Commutated Pole Inverter
}

\author{
Apollo Charalambous, Xibo Yuan, Senior Member, IEEE, and Neville McNeill
}

\begin{abstract}
Fast-switching power converters are a key enabling technology for the More Electric Aircraft (MEA), but the generated Electromagnetic Interference (EMI) poses significant challenges to the electrification effort. To meet the stringent aerospace EMI standards, passive filters are commonly employed, despite the weight and size constraints imposed by the MEA. Alternatively, the EMI source, i.e., the high $\mathrm{dv} / \mathrm{dt}$ and $\mathrm{di} / \mathrm{dt}$ slew rates, can be addressed through waveform-shaping techniques. For example, while most soft-switching converters can reduce switching loss, they do so by switching the semiconductor devices in a slower and smoother manner, resulting in the attenuation of high-frequency harmonics. This paper examines the Auxiliary Commutated Pole Inverter (ACPI) topology, and its first contribution is the attenuation of the high-frequency content of its EMI source, that is, the output voltage, in a predictable manner, through the active control of the resonant circuit. This is achieved by firstly discussing the time-domain characteristics of trapezoidal and S-shaped pulse-trains that lead to attenuated high-frequency harmonic content, and secondly, by analysing the equivalent LC circuit of the ACPI. The design of the inverter is then focused on the active control of the resonant parameters, for a predetermined and enhanced output voltage high-frequency response. The second contribution of this paper is the comparison of the EMI performance of hard switching and of three soft-switching modes, fixed-timing control, variable-timing control, and capacitive turnoffs, and how this informs important metrics like power efficiency, current stress, and implementation complexity. Lastly, the third contribution is on the trade-offs that arise when the primary design goal is enhanced EMI performance, as opposed to switching loss reduction. A 5-kW, 3-phase ACPI prototype is used for validating the high-frequency content attenuation at source. It is shown that the ACPI can achieve a 37-dB harmonic attenuation of its output voltage at $4 \mathrm{MHz}$, compared to a hard-switched inverter.
\end{abstract}

Index Terms-Auxiliary commutated pole inverter; EMI; fixed timing; frequency response; more electric aircraft; soft switching; variable timing.

\section{INTRODUCTION}

Power electronic converters play a crucial role in the electrification of vehicles and aeroplanes. For example, they are among the key enabling technologies for the More Electric Aircraft (MEA) [1], where electrical systems are increasingly adopted for replacing actuation, fuel handling and cabin air pressurisation systems traditionally powered through hydraulic,

A. Charalambous and X. Yuan are with the Department of Electrical and Electronic Engineering, University of Bristol, Bristol BS8 1UB, U.K. (e-mail: apollo.charalambous@bristol.ac.uk; xibo.yuan@bristol.ac.uk). Corresponding author: Xibo Yuan.

N. McNeill is with the Department of Electronic and Electrical Engineering, University of Strathclyde, Glasgow G1 1RD, U.K. (e-mail: neville.mcneill@strath.ac.uk). pneumatic and mechanical means. MEA offers significant potential for reducing aircraft energy consumption, and consequently, for decreasing fuel burn and emissions. With onboard electrical power generation capacity reaching 1.5 MVA for new aircrafts and future forecasts showing an upward trend with electric propulsion, aggressive power density and efficiency targets are being set for new power electronic equipment to be installed, e.g. $15 \mathrm{~kW} / \mathrm{kg}$ for power density and $95 \%+$ for efficiency. For these goals to be achieved, the switching speed is pushed higher and higher for reducing the switching loss, thus improving the efficiency and reducing the cooling requirements, e.g. with the use of wide-bandgap power devices [2]. The switching frequency is also being increased for reducing the size of the passive components, such as filtering inductors and capacitors, and for improving the power density.

However, the fast switching action of power devices such as IGBTs and MOSFETs causes high-frequency Electromagnetic Interference (EMI) [3]. This is a major disadvantage, because EMI may disrupt the operation of surrounding electronic equipment [4,5]. Furthermore, common-mode EMI (CM EMI) from voltage-source converter (VSC) based motor drives can cause motor premature winding failure, ball bearing deterioration, and motor terminal overvoltages [6-8]. The electrification effort will thus be hindered if EMI remains unchecked. This is why the aerospace industry has stringent EMI standards such as the DO-160 [9] that defines the limits of conducted EMI in the range between $150 \mathrm{kHz}$ and $152 \mathrm{MHz}$. The low-frequency EMI, i.e. from $150 \mathrm{kHz}$ to $1 \mathrm{MHz}$ is normally driven by PWM-associated harmonics and the highfrequency EMI from $1 \mathrm{MHz}$ to $152 \mathrm{MHz}$ is mainly driven by the switching transitions and the associated $\mathrm{d} v / \mathrm{dt}$ and $\mathrm{d} i / \mathrm{dt}$ rates.

Conducted EMI is usually attenuated with the help of passive filters $[3,10]$. However, filters can add a significant portion to a converter's weight and size. This negates the effort to save weight and space that is pursued by the MEA concept, and increases overall cost as well.

Instead of adding filters, the EMI emissions can be attenuated at source. For low-frequency PWM-driven EMI $(150 \mathrm{kHz}$ to $1 \mathrm{MHz})$, this can be achieved by using specialised modulation schemes like common-mode voltage cancelling [11] and random/chaotic switching [12], or even a lower switching frequency. For high-frequency switching transient-driven EMI ( $1 \mathrm{MHz}$ to $152 \mathrm{MHz}$ ), solutions include the shaping of the converter/power device waveforms such as through active gate driver control of the power devices [13], and the use of softswitching topologies. The active gate driver control method is not pursued in this work since the complexity of such circuits 
adds significant design effort, and the potential benefit of reducing both switching loss and EMI emissions is not possible.

As it is known, soft-switching topologies are commonly used for switching loss reduction by decoupling the transitioning edges of the current and voltage of the semiconductor devices $[14,15,16]$. This decoupling is usually realised with LC circuits that slow and smooth the edges in a resonant manner. When the voltage is manipulated like this, zero-voltage switching (ZVS) is performed. When the current is, zero-current switching (ZCS) is realised. Therefore, the switching transition can be effectively profiled with the $\mathrm{d} i / \mathrm{dt}$ and $\mathrm{d} v / \mathrm{dt}$ rates controlled by the resonant circuit. As such, soft-switching converters are promising in reducing switching loss as well as the high-frequency content of the switching magnitudes. This is the main reason why soft switching is pursued in this paper.

Though there is a multitude of papers discussing topologies, modulation and control of soft-switching converters, the focus is on reducing the switching loss rather than EMI attenuation. In contrast, works such as [17-22] do indeed explore the viability of reducing EMI with soft-switching converters, both in simulation and in experiment, with encouraging results. An experimental 50-kW, 3-phase auxiliary commutated pole inverter (ACPI) prototype exhibits a 10-dB drop in conducted emissions in the 3 to $5 \mathrm{MHz}$ range and a $5-\mathrm{dB}$ drop from 8 to $12 \mathrm{MHz}$ when compared to an equivalent hard-switched inverter [17]. In [21] a 70-W ZCS flyback converter exhibits a 16-dB conducted EMI attenuation in the frequency range of 5 to $20 \mathrm{MHz}$, when compared to its hard-switched counterpart. In these papers, it is recognized that some soft-switching topologies showcase an enhanced EMI performance because the $\mathrm{d} i / \mathrm{dt}$ and $\mathrm{d} v / \mathrm{dt}$ slew rates are influenced directly by the soft-switching process. However, high-frequency harmonic attenuation is treated merely as a by-product of soft switching, or at best, when EMI attenuation is actively pursued, only simulation and experimental results are presented with no insight as to how the resonant process can be actively controlled for this specific task.

In contrast, the first contribution and the focus of this paper is a method of predetermining and attenuating the highfrequency content of an EMI source through the active control of the resonant circuit. The important time-domain characteristics of pulse-trains that lead to less high-frequency content are explored, and inform the design of a soft-switching converter for exhibiting an enhanced frequency response. This is done through analytical expressions for both the highfrequency response and the resonant circuit magnitudes. For this study, the auxiliary commutated pole inverter (ACPI) is chosen, a PWM-compatible soft-switching topology that highly resembles the VSC topology. VSCs are the industry standard in variable-speed motor drives, such as the Engine Starter Motor used for jet engine start-up on MEAs [1,23]. Therefore, the ACPI can be easily employed in motor drive applications.

The developed high-frequency attenuation method is based on the active control of the ACPI's resonant circuit for reducing the harmonic content of the output voltage, which is a significant source of EMI in VSCs. At the heart of this resonant circuit lies an equivalent series LC circuit, the study of which forms the basis for the second contribution of this paper, namely the development and comparison of three ACPI soft-switching control schemes: fixed timing, variable timing, and capacitive turn-offs. The high-frequency response of these soft-switching schemes is discussed in depth, as well as how inevitably, the demand for attenuated harmonic content informs their performance regarding current stress, power efficiency, and implementation complexity. For example, while capacitive turnoffs can reduce current stress and auxiliary circuit loss by bypassing the auxiliary circuit, they generate linear output voltage edges that can potentially increase the high-frequency harmonics. Therefore in this paper, for the purpose of generating of an output voltage with only sinusoidal edges, capacitive turnoffs are initially ignored. All these trade-offs are also studied for the hard switching mode. Lastly, the third contribution of this work is on the trade-offs that arise when the ACPI is designed primarily for enhanced EMI performance, as opposed to when it is designed primarily for switching loss reduction.

This paper is structured as follows: Section II describes the operation of the ACPI. Section III presents the frequency response of trapezoidal and S-shaped pulse-trains, as the basis for an ACPI output voltage with attenuated high-frequency content. Section IV analyses the equivalent LC circuit, and explains how its resonant parameters, specifically the 'boost' currents, can actively control the duration and smoothness of the output voltage edges. Section V discusses and compares the fixed- and variable-timing control schemes with regards to implementation complexity, current stress, power loss, and crucially, output voltage edge shaping. Section VI details the ACPI design procedure for attaining an output voltage with attenuated high-frequency content. Lastly, Section VII presents the experimental results of the high-frequency attenuation, power efficiency, switching transients, and other special issues regarding the operation of the ACPI.

\section{THE AUXILIARY COMMUTATED POLE INVERTER}

\section{A. Auxiliary Resonant Pole Inverters}

This work primarily addresses high-frequency content attenuation at source for the VSC as an AC/DC rectifier, or a DC/AC inverter for motor drives in electromagnetically sensitive environments like the MEA, where it is imperative that the $\mathrm{d} v / \mathrm{dt}$ of the output voltage is reduced [24]. The 6-switch, 2level, 3-phase VSC is the standard configuration for AC/DC or DC/AC power conversion. Thus application-wise, the considered soft-switching topologies should be similar to the standard VSC. Given the focus is on the output voltage $\mathrm{d} v / \mathrm{dt}$ and profile, only ZVS inverters are considered.

The topologies with the highest degree of PWM compatibility, independent phase-leg control, and efficiency, that most resemble the VSC are the auxiliary resonant pole inverter (ARPI) family of ZVS inverters [25]. The most popular ARPIs are the coupled-inductor ZVS inverters [26-29], and the ACPI [30,31]. The coupled-inductor inverters are intended for adaptable control to any load level, and they exhibit the least amount of auxiliary circuit loss of all the ARPIs. However, they have a complicated design and high component counts. The ACPI on the other hand, might exhibit more auxiliary loss, but it has a lower component count, and its operation is simpler and more flexible. For this last reason, the ACPI is chosen as the subject of this study.

\section{B. Operation of the ACPI}

Fig. 1 shows an ACPI phase-leg, where a VSC phase-leg of main devices $S_{1} / D_{1}$ and $S_{4} / D_{4}$ is supplemented with an auxiliary branch between the DC midpoint $\mathrm{O}$ and the output node $\mathrm{A}$. This branch consists of auxiliary devices $\mathrm{S}_{\mathrm{a} 1} / \mathrm{D}_{\mathrm{a} 1}$ and $\mathrm{S}_{\mathrm{a} 4} / \mathrm{D}_{\mathrm{a} 4}$, in series with a resonant inductor $L_{\mathrm{r}} . C_{\mathrm{r} 1}$ and $C_{\mathrm{r} 4}$ are snubber capacitors that are equal in value. Three such phase-legs comprise the 3 phase ACPI, with all the auxiliary branches sharing $\mathrm{O}$. The 
clamping diodes, though not part of the topology, are essential for protecting the auxiliary devices against overvoltages.

Fig. 2 shows the waveforms of the inductor current $i_{\mathrm{Lr}}$, the phase-leg output voltage $v_{\text {pole }}$, and the switching currents $i_{\mathrm{S} 1}$ and $i_{\mathrm{S} 4}$ of the main devices, during a switch turn-on transition $\left(\mathrm{D}_{4}\right.$ to $\left.S_{1}\right)$ in Fig. 2(a), a switch turn-off transition $\left(S_{1}\right.$ to $\left.D_{4}\right)$ in Fig. 2(b), and a capacitive turn-off transition $\left(S_{1}\right.$ to $\left.D_{4}\right)$ in Fig. 2(c). The phase current $I_{\text {phase }}$ is considered positive and constant during a transition. The sub-circuits during each $v_{\text {pole }}$ transition are also shown.

The turn-on transition of Fig. 2(a) starts with $S_{\mathrm{a} 1}$ turning on first. During the $t_{\text {ramp_on }}$ interval, $i_{\mathrm{Lr}}$ ramps up and $L_{\mathrm{r}}$ accumulates energy. As $i_{\mathrm{Lr}}$ surpasses $I_{\mathrm{phase}}, i_{\mathrm{S} 4}$ reverses through $\mathrm{S}_{4}$. Then, $i_{\mathrm{Lr}}$ reaches $I_{\text {trip }}$ and $i_{\mathrm{S} 4}$ reaches $I_{\text {boost }}$. At this point, $L_{\mathrm{r}}$ starts resonating with $C_{\mathrm{r} 1}$ and $C_{\mathrm{r} 4}$. During the resonant interval $t_{\text {res_on, }} v_{\text {pole }}$ sinusoidally swings from the negative to the positive rail until it is clamped by $\mathrm{D}_{1}$. Then $i_{\mathrm{Lr}}$ starts ramping down, and the incoming $S_{1}$ is gated on. As soon as $i_{\mathrm{Lr}}$ drops below $I_{\text {phase }}, S_{1}$ starts conducting. It is seen that a resonant turn-on transition always involves a swap between the antiparallel diode and its switch, as attested by the direction reversal of $i_{\mathrm{S} 4}$ and $i_{\mathrm{S} 1}$. The swap from $D_{4}$ to $S_{4}$ allows for the controlled triggering of resonance, and a grace period is created as $\mathrm{D}_{1}$ conducts during which $\mathrm{S}_{1}$ is turned on under ZVS.

The turn-off transition of Fig. 2(b) starts with $\mathrm{S}_{\mathrm{a} 4}$ turning on first, and $i_{\mathrm{Lr}}$ ramping down in the negative direction, during the $t_{\text {ramp_off }}$ interval. Meanwhile, the outgoing switch $\mathrm{S}_{1}$ conducts the sum of $i_{\mathrm{Lr}}+I_{\text {phase. }}$. When $i_{\mathrm{Lr}}$ reaches the value of $I_{\text {boca }}$, and $i_{\mathrm{S} 1}$ the value of $I_{\text {off }}, \mathrm{S}_{1}$ is turned off and resonance commences. During the resonant interval $t_{\text {res_off, }} v_{\text {pole }}$ swings down to the negative rail, and the edge is shaped in a sinusoidal fashion. Then, the incoming $\mathrm{D}_{4}$ clamps $v_{\text {pole }}, \mathrm{S}_{4}$ is gated on, and $i_{\mathrm{Lr}}$ starts ramping up to zero. Steady state is then established with $\mathrm{D}_{4}$ conducting $I_{\text {phase. }}$

The resonant process performs ZVS turn-on and turn-off of the main devices, and ZCS turn-on and natural turn-off of the auxiliary ones. The voltage and the current are decoupled, with resonance slowing down $v_{\text {pole }}$ and shaping it in a smooth, sinusoidal manner. Hence, the ACPI can reduce switching loss, while profiling the $\mathrm{d} v / \mathrm{dt}$ of $v_{\text {pole. }}$. However, with hard switching no auxiliary circuit power loss exists [25,31]. Also, soft switching increases the current stress on the main devices, especially during the turn-off transition, because of the inductor current components $I_{\text {boost }}$ and $I_{\mathrm{off}}$ that are imposed on $i_{\mathrm{S} 1}$ and $i_{\mathrm{S} 4}$. Hard switching though cannot perform waveform shaping and smoothing, like soft switching can.

The main device current stress caused by the resonant turnoff transition can be avoided when a capacitive turn-off takes place, as seen in Fig. 2(c). Simply, $I_{\text {phase }}$ alone discharges $C_{\mathrm{r} 4}$ and charges $C_{\mathrm{r} 1}$, and $v_{\text {pole }}$ transitions in a linear rather than a resonant manner, like a lossless turn-off snubber [14,32]. The auxiliary

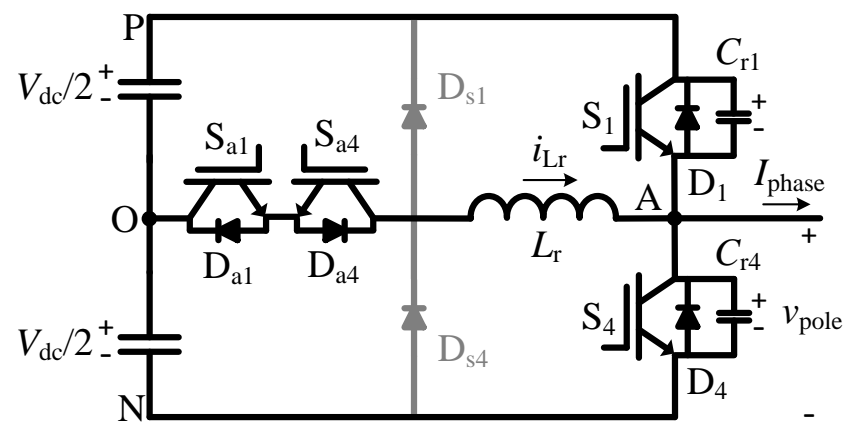

Fig. 1. The circuit schematic of the ACPI phase-leg. circuit is not utilized, which avoids the auxiliary loss and the extra current stress on the main devices. However, a capacitive turn-off is possible only at sufficiently high $I_{\text {phase }}$ levels. Otherwise it will last too long, causing problems such as a current spike in the incoming device [33,34], and the drop of pulses if the PWM time constraints are violated.

In summary, the ACPI performs three kinds of transition: resonant switch turn-ons that occur during the entire fundamental cycle $T_{1}$, resonant switch turn-offs, and capacitive switch turn-offs. Resonant turn-off transitions can be utilized during the entirety of $T_{1}$, or at high $I_{\text {phase }}$ levels, they can be replaced with capacitive ones. The way that each kind of transition occurs, influences the $v_{\text {pole }}$ high-frequency, as well as the overall power loss and current stress of the ACPI.

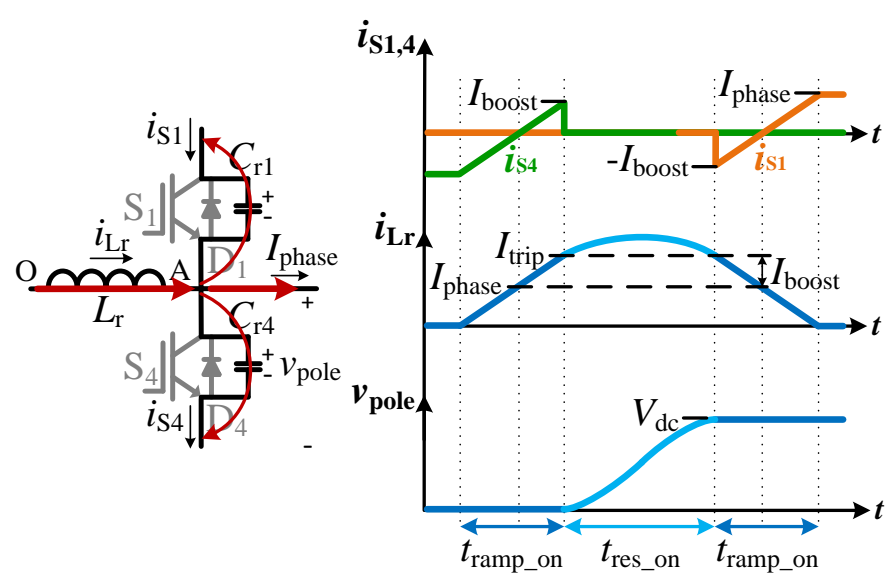

(a)

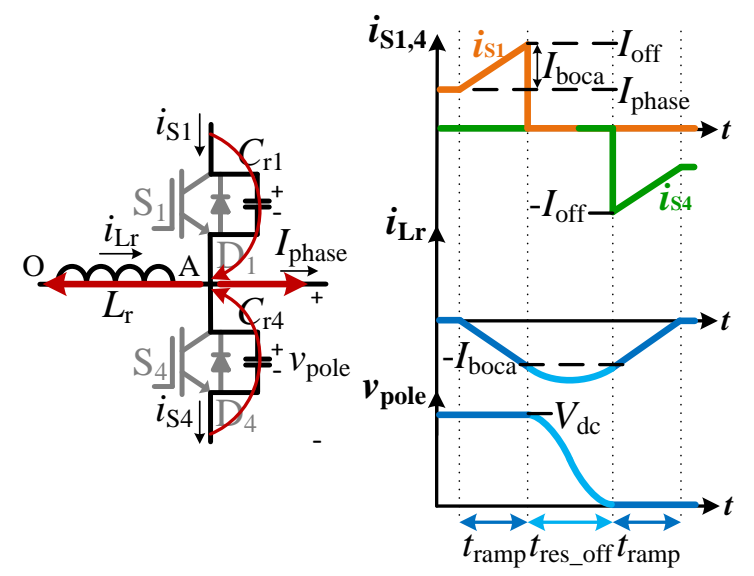

(b)

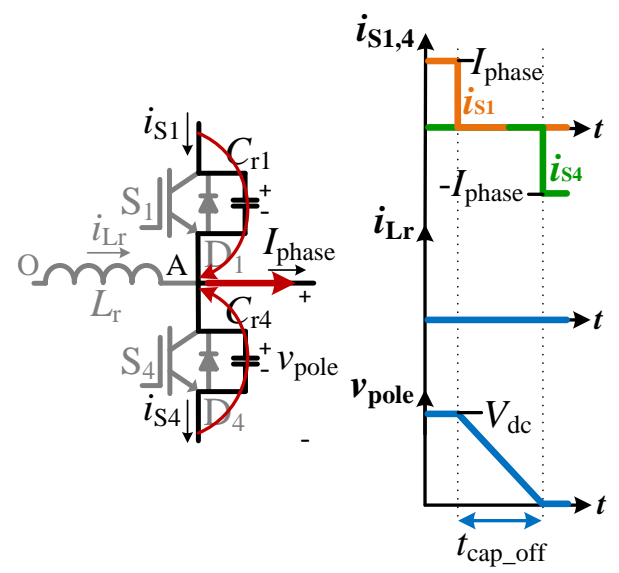

(c)

Fig. 2. Inductor current $i_{\mathrm{Lr}}$, output voltage $v_{\text {pole }}$, switching currents $i_{\mathrm{S} 1}$ and $i_{\mathrm{S} 4}$, and corresponding circuits during transitions of $\mathrm{S}_{1}$ : (a) a resonant turn-on, (b) a resonant turn-off, and (c) a capacitive turn-off, when $I_{\text {phase }}>0$. 


\section{FREQUENCY RESPONSE OF PULSE-TRAINS}

The frequency response of pulse-trains commonly found in power converters is reviewed in this section, as it is the foundational theory for designing the ACPI for high-frequency attenuation purposes. Here, the frequency response of the trapezoidal, the S-shaped, and the sinusoidal pulse-train is briefly discussed. It is assumed that a hard-switched converter has trapezoidal output voltage pulses, and that a soft-switching converter has S-shaped or sinusoidal voltage pulses.

Fig. 3 shows symmetrical trapezoidal and S-shaped pulses of a fixed pulse-width that are successively differentiated, until an impulse train appears $[3,35,36]$. This graphical representation serves the intuitive interpretation of their high-frequency content: the higher the derivative where the impulse train appears, the lesser the high-frequency harmonic content, and the larger the amplitude of the impulses, the higher that content is [36]. Also featured are the parameters of the pulse-width $\tau$, the rise/fall time $t_{\mathrm{r}}$, and the first-derivative rise time $t_{\mathrm{r}(\mathrm{dv} / \mathrm{dt})}$ of the corners of the S-shaped pulse.

The derivative order where the impulse train appears, corresponds to the number of corner frequencies on each symmetrical pulse-train's spectral envelope. This way, the trapezoidal spectral envelope has two corner frequencies as in (1) and (2): $f_{\mathrm{c} 1}$ between the envelope slopes of 0 to $-20 \mathrm{~dB} / \mathrm{dec}$, and $f_{\mathrm{c} 2}$ between -20 to $-40 \mathrm{~dB} / \mathrm{dec}$. The S-shaped envelope has three corner frequencies: while $f_{\mathrm{c} 1}$ is the same, the $t_{\mathrm{r}(\mathrm{dv} / \mathrm{dt})}$ parameter defines $f_{\mathrm{c} 2}$ and a third frequency $f_{\mathrm{c} 3}$, as in (3) and (4). The smooth corners of the S-shaped edges and the introduction of $f_{\mathrm{c} 3}$ marks a slope change from -40 to $-60 \mathrm{~dB} / \mathrm{dec}$. Hence, the pulse-width $\tau$ primarily influences the lower end of the spectrum, and $t_{\mathrm{r}}$ and $t_{\mathrm{r}(\mathrm{dv} / \mathrm{dt})}$ the higher end $[3,35,36]$.

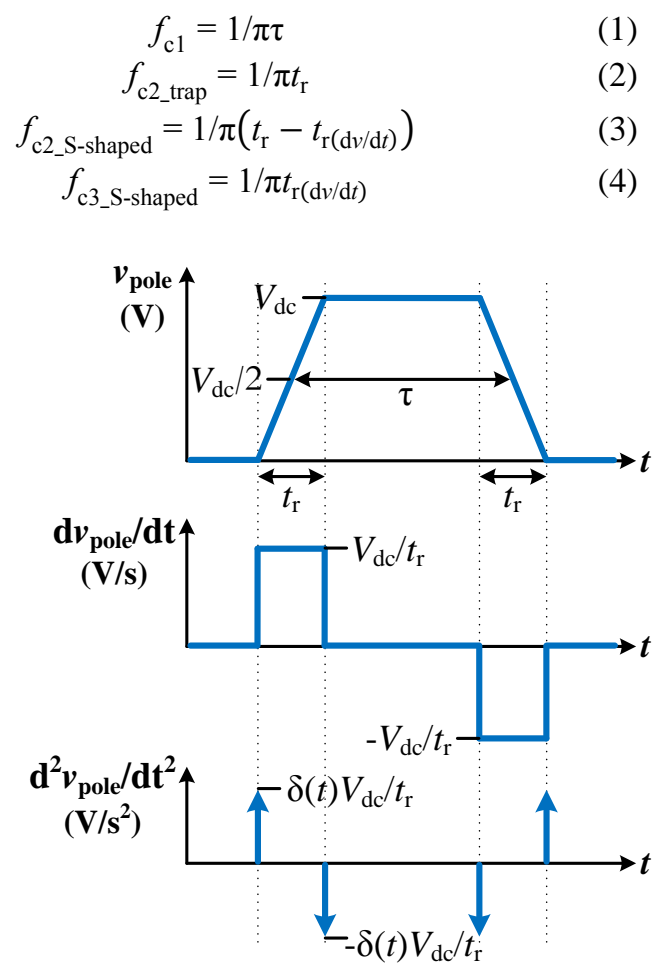

(a)
As described in Section II, if only resonant transitions are realised, the output voltage of the ACPI will consist of pulses with sinusoidal edges. If these pulses are assumed as symmetrical and of a fixed pulse-width, the $v_{\text {pole }}$ pulse-train will only have two corner frequencies: the $f_{\mathrm{c} 1}$ of (1) and an $f_{\mathrm{c} 2}$ that marks a direct slope change from -20 to $-60 \mathrm{~dB} / \mathrm{dec}$ [37]. However, the successive time-derivative method cannot be applied to sinusoidal edges, as they can be infinitely differentiated, yet they can be approximated as S-shaped, with a small error of about $4 \mathrm{~dB}$, by defining that $t_{\mathrm{r}}=2 \cdot t_{\mathrm{r}(\mathrm{dv} / \mathrm{dt})}$ [36]. The result is an S-shaped pulse-train where $f_{\mathrm{c} 2}=f_{\mathrm{c} 3}$. This special Sshaped pulse maintains the sinusoidal property of the direct slope change to $-60 \mathrm{~dB} / \mathrm{dec}$. Fig. 4 shows a simulation of trapezoidal, S-shaped, and 'sinusoidal' spectra of duty cycle $d=0.5$, switching frequency $f_{\mathrm{s}}=20 \mathrm{kHz}$, and symmetrical edges. It is clearly seen that the $-60 \mathrm{~dB} / \mathrm{dec}$ slope in the $\mathrm{S}$-shaped and sinusoidal spectra results in less high-frequency harmonic content than the trapezoidal spectrum.

In Fig. $4, d$ is fixed, although the $v_{\text {pole }}$ pulse-train of the ACPI is modulated with Sinusoidal PWM (SPWM), where $d$ varies during $T_{1}$ and is dictated by the amplitude modulation index $m_{\mathrm{A}}$ [38]. While in this case it is difficult to estimate the harmonic content, it will always be less than the pulse-train of a fixed $d=0.5$, i.e., of $m_{\mathrm{A}}=0$ [39]. As such, the envelope under $m_{\mathrm{A}}=0$ is always the worst case for the envelope under $m_{\mathrm{A}} \neq 0$, in the entire frequency range. If the pulses have identical edges between pulse-trains, the corner frequencies described in (2), (3), and (4) will not be affected.

This analysis highlights that the edges of an $\mathrm{S}$-shaped/sinusoidal pulse can be manipulated in a flexible way, through their duration $\left(t_{\mathrm{r}}\right)$ and shape $\left(t_{\mathrm{r}(\mathrm{dv} / \mathrm{dt})}\right)$, that is, their smooth corners. For the reasons discussed above, the frequency response of the sinusoidal $v_{\text {pole }}$ pulses of the ACPI is treated as S-shaped. Based on this analysis, the ACPI resonance can be manipulated in a way that shapes the edges of the output voltage for an improved and more predetermined high-frequency attenuation.

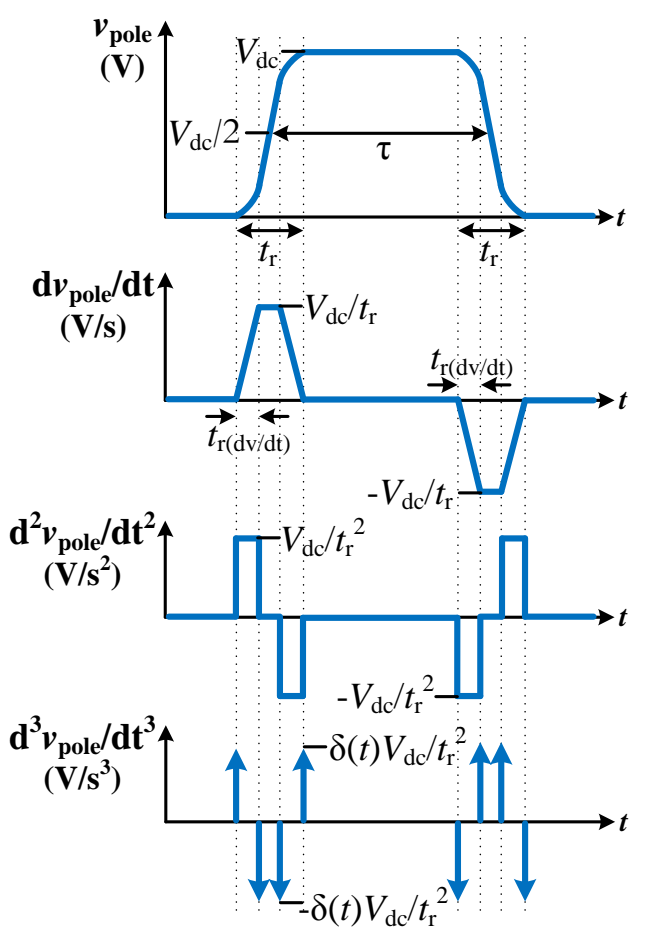

(b)

Fig. 3. Successive differentiations of (a) a symmetrical trapezoidal pulse, and of (b) a symmetrical S-shaped pulse. 


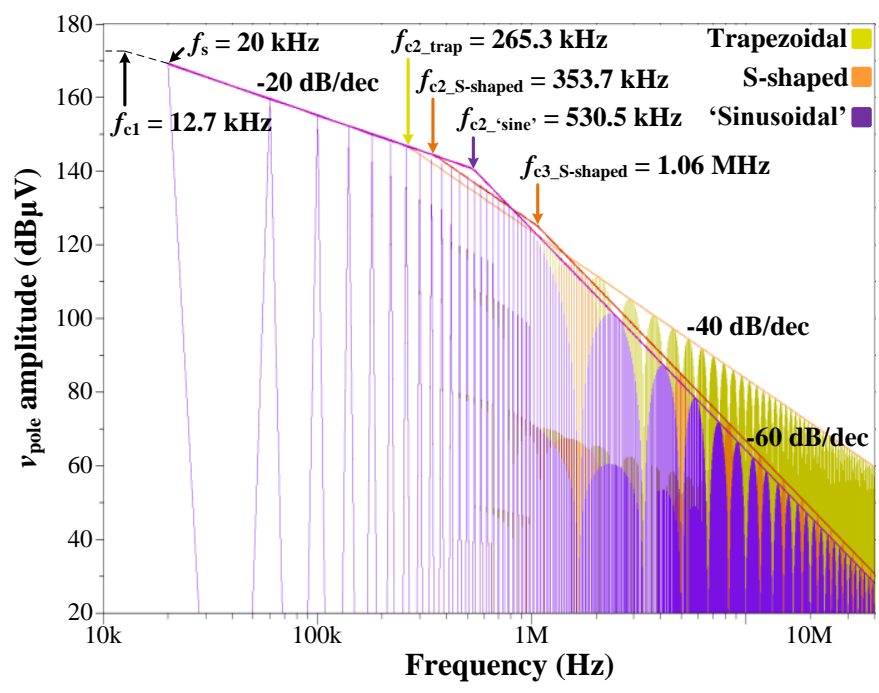

Fig. 4. Trapezoidal, S-shaped, and 'sinusoidal' spectra with $V_{\mathrm{dc}}=450 \mathrm{~V}$, $f_{\mathrm{s}}=20 \mathrm{kHz}, \tau=25 \mu \mathrm{s}, d=0.5, t_{\mathrm{r}}=1.2 \mu \mathrm{s}, t_{\mathrm{r}(\mathrm{dv} / \mathrm{dt})}=300 \mathrm{~ns}$ for the S-shaped edges, and $t_{\mathrm{r}(\mathrm{dv} / \mathrm{dt})}=600 \mathrm{~ns}$ for the 'sinusoidal' edges.

\section{THE EQUivalent SERIES LC CIRCUIT}

In order to shape the edges of the $v_{\text {pole }}$ pulses for an optimum frequency response, the ACPI resonant process has to be studied. Capacitive turn-offs are not considered in this section, since it is desirable that the $v_{\text {pole }}$ edges be sinusoidal only.

\section{A. The Free Oscillation of the Equivalent Circuit and the Quasi-resonant Action of the ACPI}

Any resonant transition starts with a set of initial conditions that are dictated by its type (turn-on or turn-off), and by the level and polarity of $I_{\text {phase. This process is represented as an equivalent }}$ series LC circuit, whose solution is the inductor current $i_{\mathrm{Lr}}$ and the output voltage $v_{\text {pole }}$. Figs. 5(a) and (b) illustrate the LC circuits and their initial conditions for the turn-on and turn-off transitions, when $I_{\text {phase }}$ is positive. These circuits are derived directly from the circuits in Fig. 2. It is seen that resonance is driven by half the DC-link voltage $V_{\mathrm{dc}} / 2$, while $I_{\text {phase }}$ acts as a parallel DC load. The $2 C_{\mathrm{r}}$ capacitor between the output node A and the negative rail represents the two snubber capacitors.

The step response of the LC circuits results in $i_{\mathrm{Lr}}$ and $v_{\text {pole }}$ oscillating sinusoidally with a resonant period $T_{0}$, between a peak and a valley. The resonant energy available in the circuit depends on the level of $V_{\mathrm{dc}}$, the properties of $I_{\text {phase }}$, and the initial conditions. If the energy increases by raising the $i_{\mathrm{Lr}}(0)$ initial condition for example, the sinusoids will oscillate to more extreme peaks and valleys but with the same angular frequency $\omega_{0}$. This is presented in Fig. 5(c), where two $v_{\text {pole }}$ waveforms oscillate freely in response to the energy present in the circuit. Waveform 1 oscillates to more extreme values than waveform 2 , due to more energy in the circuit, while $\omega_{0}$ is maintained.
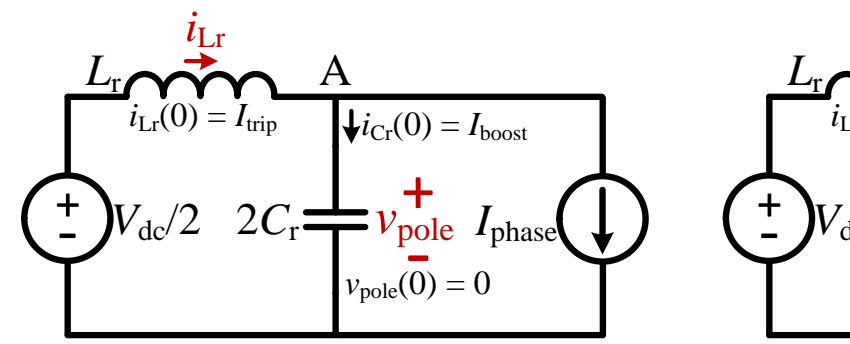
$i_{\mathrm{Lr}}$

(a)
It is important to note however, that the ACPI is a quasiresonant topology. Resonance only happens when a main switch turns on or off. This contrasts with how other resonant DC-link inverters operate, where the ZVS transitions are realized by a continuously-resonating DC-link voltage. The basic resonant DC-link inverter [15,40] and its derivatives [41] operate in this manner. In the case of the ACPI however, only part of the free oscillation is experienced in the resonant circuit, as $v_{\text {pole }}$ swings between the two DC rail values of (ideally) 0 and $V_{\mathrm{dc}}$, during the resonant interval $t_{\text {res }}$. The two DC rail values are not necessarily at the extremes of the free oscillation, however. As seen in Fig. 5(c), the two $v_{\text {pole }}$ waveforms oscillate between 0 and $V_{\mathrm{dc}}$, only during the $t_{\text {res }}$ intervals. Waveform 1 has a larger amplitude, meaning that its free oscillation reaches more extreme peaks and valleys than waveform 2 . Hence, the $t_{\text {res }}$ interval of waveform 1 is shorter, and the $\mathrm{d} v / \mathrm{dt}$ gradient is larger. The reason for this behaviour is that the amount of energy present in the resonant circuit for waveform 1 is larger than for waveform 2 . Consequently, the energy in the resonant circuit dictates the duration $\left(t_{\text {res }}\right)$ and the shape of the $v_{\text {pole }}$ edge between the DC rail values, impacting the high-frequency response.

\section{B. Boost and Trip Currents}

The main difference between the turn-on and the turn-off transitions is the role of $I_{\text {phase. }}$. During the turn-on transition of Fig. 5(a), $i_{\text {Lr }}$ flows into A to charge the capacitor, while $I_{\text {phase }}$ flows out of it as to discharge it, thus inhibiting resonance. When the $i_{\mathrm{Lr}}(0)$ condition is kept constant, less energy is available to complete resonance, as $I_{\text {phase }}$ increases during the fundamental cycle $T_{1}$. Conversely, in Fig. 5(b), both $i_{\text {Lr }}$ and $I_{\text {phase }}$ flow out of A and discharge the capacitor together. If $i_{\mathrm{Lr}}(0)$ is kept constant, more energy is put into the circuit as $I_{\text {phase }}$ increases. Under these conditions, the $v_{\text {pole }}$ edge swings faster between the DC rails and becomes steeper during the turn-off transitions. The opposite trend applies for turn-ons.

A distinction concerning the initial conditions of the currents is necessary at this point. $I_{\text {boost }}$ and $I_{\text {off }}$, the currents denoted as the initial conditions $i_{\mathrm{Cr}}(0)$ of the capacitor current in Figs. 5(a) and (b), are the values reached by the outgoing switch current right before resonance starts, as seen in Figs 2(a) and (b). $I_{\text {boost }}$ and $I_{\text {off }}$ are termed here as the boost currents and are controlled by the ramp time intervals $t_{\text {ramp. }}$. The $i_{\mathrm{Lr}}(0)$ conditions of a given turn-on or turn-off transition are also controlled by $t_{\text {ramp. In Figs. }}$ 2 (a) and (b), $i_{\mathrm{Lr}}$ ramps up to the values of $I_{\text {trip }}$ and $I_{\text {boca }}$, which are termed here as the trip currents. Lastly, the relationship between the boost and the trip currents involves $I_{\text {phase }}$, as seen in Fig. 5 .

The trip currents must be large enough to overcome the power loss incurred by the parasitic resistance of the auxiliary circuit $[30,42]$. The voltage that drives the ACPI resonance is always $V_{\mathrm{dc}} / 2$, and it does not provide enough energy to the

Fig. 5. Equivalent series LC circuit of (a) a switch turn-on transition, (b) a switch turn-off transition when $I_{\text {phase }}>0$, and (c) ACPI quasi-resonant action applied to two $v_{\text {pole }}$ sinusoids of the same frequency but of different amplitudes. 
resonant circuit for overcoming that power loss. Thus, additional inductor current is required for compensating the loss, and allowing $v_{\text {pole }}$ to swing fully to the opposite rail and achieve ZVS. Conversely, the coupled-inductor inverters [26-29] raise the driving voltage to more than $V_{\mathrm{dc}} / 2$, allowing $i_{\mathrm{Lr}}$ to reach lower trip values. Customarily, $I_{\text {trip }}=I_{\text {phase }}$ is set for turn-ons, meaning that $I_{\text {boost }}=0$. This reduces the auxiliary circuit loss. However, these inverters suffer from high component counts and design complexity. While the boost currents in the ACPI increase the auxiliary circuit loss and the main device current stress, they allow flexibility in controlling resonance and the duration of the resonant intervals $t_{\text {res. }}$ This is crucial, since the $t_{\text {res }}$ intervals are the actual rise and fall times of $v_{\text {pole. As such, this work }}$ investigates the ACPI for high-frequency content attenuation. Also, interesting trade-offs arise between the power efficiency and the output voltage high-frequency attenuation, due to the existence of the boost currents and the way they are controlled.

\section{Analytical Expressions of the Equivalent Circuits}

The equivalent circuits are described by ordinary differential equations that yield sinusoidal $i_{\mathrm{Lr}}$ and $v_{\text {pole }}$ expressions, as in (5) and (6) for turn-ons, and (7) and (8) for turn-offs. A positive $I_{\text {phase }}$ and no auxiliary circuit loss are assumed. Also during resonance, $i_{\mathrm{Lr}}$ is bounded by its trip values $I_{\text {trip }}$ and $I_{\text {boca }}$, and $v_{\text {pole }}$ swings between 0 and $V_{\text {dc }}$, like in Fig. 2.

$$
\begin{gathered}
i_{\mathrm{Lr}}=I_{\text {phase }}+I_{\text {boost }} \cos \left(\omega_{0} t\right)+\frac{V_{\mathrm{dc}} / 2}{Z_{0}} \sin \left(\omega_{0} t\right) \\
v_{\text {pole }}=\frac{V_{\mathrm{dc}}}{2}\left[1-\cos \left(\omega_{0} t\right)\right]+Z_{0} I_{\text {boost }} \sin \left(\omega_{0} t\right) \\
i_{\mathrm{Lr}}=I_{\text {phase }}-I_{\text {off }} \cos \left(\omega_{0} t\right)-\frac{V_{\mathrm{dc}} / 2}{Z_{0}} \sin \left(\omega_{0} t\right) \\
v_{\text {pole }}=\frac{V_{\mathrm{dc}}}{2}\left[1+\cos \left(\omega_{0} t\right)\right]-Z_{0} I_{\text {off }} \sin \left(\omega_{0} t\right)
\end{gathered}
$$

$Z_{0}$ and $\omega_{0}$ are the resonant impedance and the resonant frequency, respectively, and depend on the values of the resonant components $L_{\mathrm{r}}$ and $C_{\mathrm{r}}$, as in (9) and (10). $Z_{0}$ and $\omega_{0}$ are valid for both equivalent LC circuits.

$$
\begin{gathered}
Z_{0}=\sqrt{L_{\mathrm{r}} / 2 C_{\mathrm{r}}} \\
\omega_{0}=1 / \sqrt{2 L_{\mathrm{r}} C_{\mathrm{r}}}
\end{gathered}
$$

Solving (5) for $i_{\mathrm{Lr}}=I_{\text {trip }}$ and (7) for $i_{\mathrm{Lr}}=\left|I_{\text {bocal }}\right|$, yields the expression for $t_{\text {res_on }}$ in (11), and for $t_{\text {res_off }}$ in (12).

$$
\begin{aligned}
& t_{\text {res_on }}=\frac{2}{\omega_{0}} \tan ^{-1}\left[\frac{V_{\mathrm{dc}} / 2}{Z_{0}\left(I_{\text {trip }}-I_{\text {phase }}\right)}\right]=\frac{2}{\omega_{0}} \tan ^{-1}\left(\frac{V_{\mathrm{dc}} / 2}{Z_{0} I_{\text {boost }}}\right) \\
& t_{\text {res_off }}=\frac{2}{\omega_{0}} \tan ^{-1}\left[\frac{V_{\text {dc }} / 2}{Z_{0}\left(\left|I_{\text {boca }}\right|+I_{\text {phase }}\right)}\right]=\frac{2}{\omega_{0}} \tan ^{-1}\left(\frac{V_{\mathrm{dc}} / 2}{Z_{0} I_{\text {off }}}\right)
\end{aligned}
$$

It is notable that the boost currents $I_{\text {boost }}$ and $I_{\text {off }}$ appear in (5), (6), (7), (8), (11) and (12). Hence, for given circuit conditions $V_{\mathrm{dc}}$ and $I_{\text {phase }}$, and for given $L_{\mathrm{r}}$ and $C_{\mathrm{r}}$ values, the boost currents affect the resonant magnitudes and the resonant intervals. By properly controlling the boost currents, $v_{\text {pole }}$ can be profiled with slow and smooth edges for reduced high-frequency content, in a predetermined way, and in line with the theory of Section III.

\section{FIXED-TIMING AND VARIABLE-TIMING CONTROL}

The thorough analysis of the equivalent LC circuit serves for deriving the ACPI control schemes. The two classic ACPI schemes are fixed-timing and variable-timing control [33]. In $[43,44]$ these terms refer to the inductor current ramp interval $t_{\text {ramp. If these intervals are fixed throughout the fundamental }}$ cycle $T_{1}$, fixed-timing control is realised. If they vary, variabletiming control is realised. This terminology has also been applied to the conduction period $t_{\text {aux_sw }}$ of the auxiliary devices [33]. Regardless of the parameter they refer to, these schemes differ in ease of implementation, current stress, power loss, and most importantly, in high-frequency harmonic attenuation.

Fixed timing is simple to implement. The $t_{\text {ramp }}$ intervals described by (13) and (14) are fixed and are manually set into the control algorithm. This means that for given $L_{\mathrm{r}}$ and $V_{\mathrm{dc}}$ values, the trip currents $I_{\text {trip }}$ and $I_{\text {boca }}$ are fixed throughout $T_{1}$.

$$
\begin{aligned}
& t_{\text {ramp_on }}=\frac{L_{\mathrm{r}} I_{\text {trip }}}{V_{\mathrm{dc}} / 2}=\frac{L_{\mathrm{r}}\left(I_{\text {boost }}+I_{\text {phase }}\right)}{V_{\mathrm{dc}} / 2} \\
& t_{\text {ramp_off }}=\frac{L_{\mathrm{r}}\left|I_{\text {boca }}\right|}{V_{\mathrm{dc}} / 2}=\frac{L_{\mathrm{r}}\left(I_{\mathrm{off}}-I_{\text {phase }}\right)}{V_{\mathrm{dc}} / 2}
\end{aligned}
$$

Moreover, the control algorithm uses the $I_{\text {phase }}$ polarity information for selecting the appropriate auxiliary switch that for a given resonant transition: $S_{\mathrm{a} 1}$ helps with turn-ons and $S_{\mathrm{a} 4}$ with turn-offs, when $I_{\text {phase }}$ is positive. When $I_{\text {phase }}$ is negative, the auxiliary switches swap roles. However, this scheme can be greatly simplified if the $t_{\text {ramp }}$ intervals are set equal, i.e., $t_{\text {ramp_on }}=$

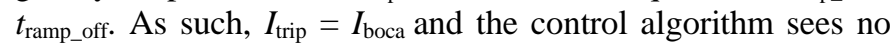
distinction between a turn-on and a turn-off transition. This simplification results in no current and voltage sensing for the purpose of resonance.

The simple fixed-timing scheme comes at the expense of increased current stress and power loss [33]. Firstly, the $i_{\text {Lr }}$ pulse currents are unnecessarily high, as seen in Fig. 6(a). This means that throughout $T_{1}$, the current stress in the auxiliary devices is severe due to the large $i_{\mathrm{Lr}}$ peaks. Secondly, the RMS value $I_{\mathrm{Lr} \_r m s}$ of $i_{\text {Lr }}$ is large, causing high auxiliary circuit loss. Thirdly, the main device current stress increases, especially during the resonant turn-offs when $I_{\text {phase }}$ is near its peak value $I_{\text {phase_pk }}$. According to $I_{\text {off }}=I_{\text {boca }}+I_{\text {phase }}, I_{\text {off }}$ becomes excessively large when $I_{\text {phase_pk }}$ is reached, as $I_{\text {boca }}$ is fixed throughout $T_{1}$. Hence, the peaks of the main switching currents $i_{\mathrm{S} 1}$ and $i_{\mathrm{S} 4}$ in Fig. 2(b),

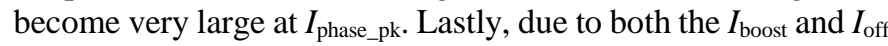
contributions in $i_{\mathrm{S} 1}$ and $i_{\mathrm{S} 4}$, the conduction losses of the main devices are increased.

Variable-timing control reduces current stress and power loss by varying the $t_{\text {ramp }}$ intervals [33], which builds the trip currents and the overall $i_{\text {Lr }}$ pulses according to the $I_{\text {phase }}$ level. As seen in Fig. 6(b), the turn-on pulses are initially small, then turn larger as more $i_{\text {Lr }}$ contribution is required at higher $I_{\text {phase }}$ levels. The reverse trend holds for the turn-off pulses, since, as explained in Section IV, less $i_{\text {Lr }}$ contribution is required as $I_{\text {phase }}$ increases. Thus, $I_{L_{-} \_r m s}$ and the peaks in the main device currents are reduced. However, this optimized scheme is more complex, because the $V_{\mathrm{dc}}$ value, and the $I_{\text {phase }}$ level and polarity information are required for the online calculation of the $t_{\text {ramp }}$ intervals of (13) and (14).

Most importantly, the fixed- and variable-timing schemes have a profound influence on how resonance transpires, which

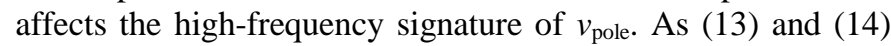
indicate, under fixed timing, the boost currents $I_{\text {boost }}$ and $I_{\text {off }}$ vary throughout $T_{1}$, since the trip currents $I_{\text {trip }}$ and $I_{\text {boca }}$ are fixed. In turn, the resonant intervals $t_{\text {res_on }}$ and $t_{\text {res_off }}$ vary during $T_{1}$, according to (11) and (12). As explained in Section IV, when the trip currents are fixed, the resonant energy increases for turn-offs and decreases for turn-ons, as $I_{\text {phase increases. This erratic }}$ provision of energy means that from one switching cycle to the next, each $v_{\text {pole }}$ pulse will be dissimilar to its adjacent ones in 
terms of edge duration and shape. As a result, the frequency response will not be as predictable as the spectra of Fig. 4.

In contrast, under variable-timing control, the varying $t_{\text {ramp }}$ intervals of (13) and (14) mean that they can be controlled in a way that $I_{\text {boost }}$ and $I_{\text {off }}$ become fixed during $T_{1}$. In turn, the resonant intervals will become fixed throughout $T_{1}$, as dictated by (11) and (12). This means that the energy put into resonance is more consistent as $I_{\text {phase }}$ varies. The $v_{\text {pole }}$ edges will then be shaped in a consistent manner from one switching cycle to the next, and the frequency response can become more predictable. This is preferable for high-frequency harmonic attenuation. The $v_{\text {pole }}$ pulses generated by each scheme are shown in Fig. 7. In Fig. 7(a) it is seen that fixed timing produces edges that change from one switching cycle to the next, whereas in Fig. 7(b) the edges are consistent in duration and shape under variable timing.

In addition, the variable-timing control algorithm can be set up in such a way that the boost currents are both fixed and equal during the fundamental cycle, i.e., $I_{\text {boost }}=I_{\text {off. }}$ Then, as dictated by (11) and (12), the resonant intervals will also be equal, i.e., $t_{\text {res_on }}=t_{\text {res_off. }}$ The consequence of this condition is the generation of $v_{\text {pole }}$ pulses with the same rising and falling edges. In other words, the pulses can become symmetrical and the highfrequency response of the output voltage will be similar to the one of a sinusoidal-edge pulse-train, as simulated in Fig. 4.

Conclusively, proper boost current control is essential to the active control of the ACPI resonant magnitudes [45,46], with the overall loss and current stress depending on the properties of the inductor current $i_{\mathrm{Lr}}$, and the high-frequency attenuation capability depending on the edge properties of the output voltage

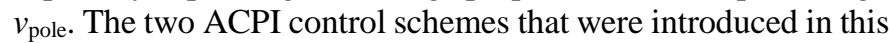
section influence the way the boost currents inject energy into the resonant circuit during each switching cycle, and therefore have a direct effect on the resonant magnitudes.

\section{DESIGNING THE ACPI FOR HIGH-FREQUENCY CONTENT ATTENUATION}

In this section, the design procedure for the experimental 3phase ACPI prototype is presented. The timing constraints imposed by the soft-switching action are defined, and the boost current condition for symmetrical $v_{\text {pole }}$ pulses is introduced, along with its advantages and disadvantages. Lastly, capacitive switch turn-offs are also considered.

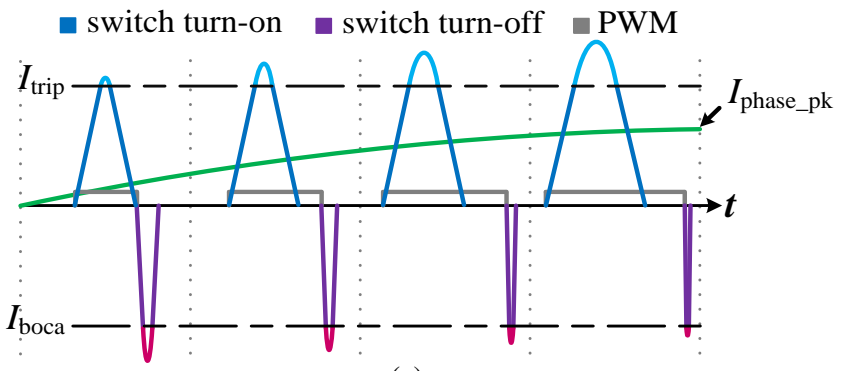

(a)

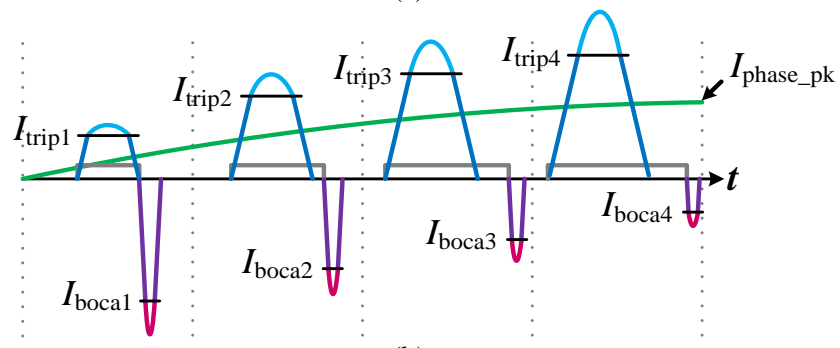

(b)

Fig. 6. Comparison of $i_{\mathrm{Lr}}$ pulses with (a) fixed-timing, and (b) variable-timing control during a fundamental quarter-cycle.

\section{A. Timing Constraints}

Successful soft switching depends on two factors. Firstly, $v_{\text {pole }}$ must swing fully to the opposite rail. This achieves ZVS, and $v_{\text {pole }}$ edges that are completely shaped by resonance. If this does not happen, then an abrupt jump of high $\mathrm{d} v / \mathrm{dt}$ will be experienced across the large snubber capacitors, and current spikes that are harmful to the main devices might appear.

Secondly, $i_{\mathrm{Lr}}$ must diminish to zero after a transition has ended and steady state is reached. If the auxiliary switch is turned off before $i_{\mathrm{Lr}}$ becomes zero, the slew rate $\mathrm{d} i_{\mathrm{Lr}} / \mathrm{dt}$ of the inductor current will sharply increase, causing an overvoltage in the auxiliary branch. The clamping diodes in Fig. 1 will protect the auxiliary devices against this overvoltage, but the control should avoid this scenario, as the voltage spikes and the sharp $\mathrm{d} i_{\mathrm{Lr}} / \mathrm{dt}$ can be secondary sources of EMI. To avoid this possibility, the conduction period of the auxiliary devices $t_{\text {aux_sw }}$ is the first parameter to be set, and it is common to both the fixedtiming and the variable-timing schemes. The $i_{\text {Lr }}$ pulse-width, denoted as $t_{\text {aux }}$, must be fully accommodated by $t_{\text {aux_sw }}$ during any transition of $T_{1}$. As such, the $t_{\text {aux_sw }}$ interval can be forced to vary along with $t_{\text {aux }}$, but that would involve sensing the actual $i_{\mathrm{Lr}}$ pulses, which is a costly and difficult thing to do.

Instead, a fixed $t_{\text {aux_sw }}$ is selected, a value that lies in the range

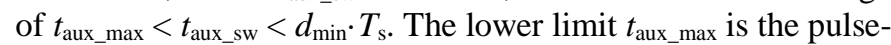
width of the tallest and widest $i_{\text {Lr }}$ pulse that appears during $T_{1}$. This specific pulse is built during the turn-on transitions at the phase current peaks $\pm I_{\text {phase_pk }}$, and its width is defined as $t_{\text {aux_max }}$ $=2 t_{\text {ramp }}+t_{\text {res_on_max }}$ under fixed-timing control, and as $t_{\text {aux_max }}=$ $2 t_{\text {ramp_on_max }}+t_{\text {res }}$ under variable-timing control. The upper boundary $d_{\min } \cdot T_{\mathrm{s}}$ is the minimum pulse-width of the SPWM signal, with $d_{\min }$ the minimum duty cycle and $T_{\mathrm{s}}$ the switching period, and setting it as the upper boundary ensures that the auxiliary switch is gated off before the next transition begins. This boundary $T_{\mathrm{s}}$ shrinks if the switching frequency $f_{\mathrm{s}}$ and/or the modulation index $m_{\mathrm{A}}$ are increased. The modulation-imposed constraints on soft switching are more stringent since the transition times usually last an order of magnitude longer than with hard switching.

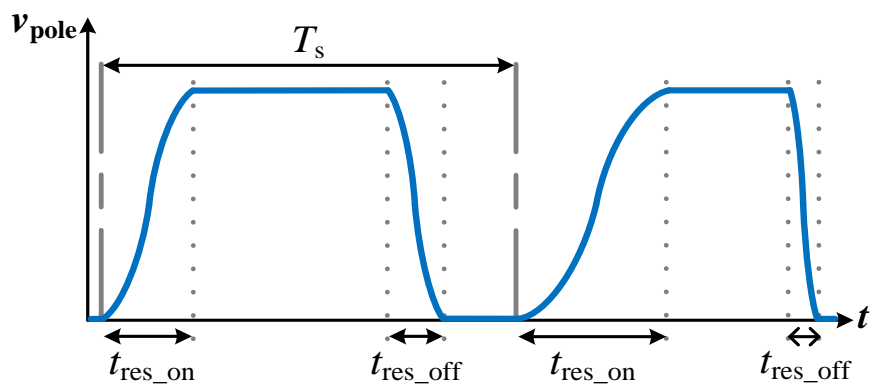

(a)

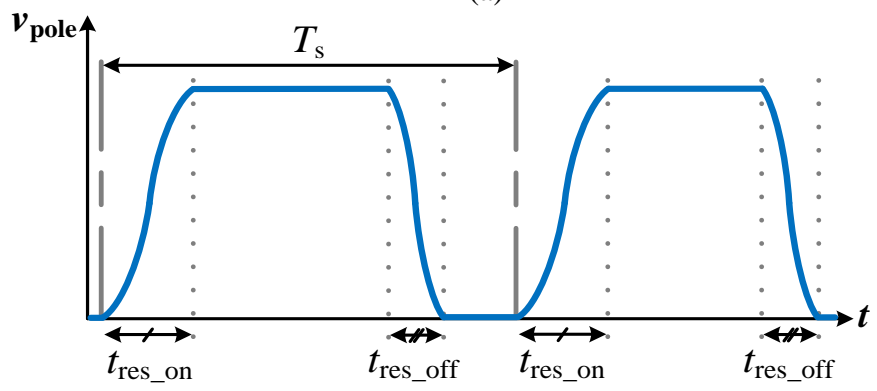

(b)

Fig. 7. Part of the $v_{\text {pole }}$ pulse-train under (a) fixed-timing, and (b) variabletiming control when $I_{\text {phase }}>0$. 


\section{B. The Boost Current Condition}

As discussed in Sections IV and V, the boost currents dictate the amount of energy provided to the resonant circuit per switching cycle. This in turn affects $i_{\text {Lr }}$ and $v_{\text {pole }}$, as well as $t_{\text {res_on }}$

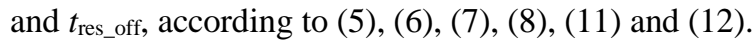

The main focus of the design procedure is the attenuation of the high-frequency content of $v_{\text {pole. Essentially, its frequency }}$ response should be optimized compared to an equivalent hardswitched inverter, by exhibiting a corner frequency $f_{\mathrm{c} 2}$ early in the spectrum and a $-60 \mathrm{~dB} / \mathrm{dec}$ roll-off at high frequencies, as discussed in Section III. Hence, the resonant intervals are demanded to be at least 5 or 10 times longer than hard switching.

Theoretically, an optimised $v_{\text {pole }}$ frequency response can be achieved more easily if it is demanded that $t_{\text {res_on }}=t_{\text {res_off, }}$, as this will ideally generate a train of symmetrical pulses, and a frequency response that is as predictable as possible. This way, only one $t_{\text {res }}$ value will have to be selected. This can only happen with variable-timing control, which is why it is the first control scheme to be designed. Symmetry in the $v_{\text {pole }}$ pulses can theoretically be achieved by demanding that:

$$
I_{\text {boost }}=I_{\text {off }}=I_{\text {phase_pk }}
$$

This boost current condition given in (15) means that during the longest turn-on transition at $I_{\text {phase_pk, }}$ the maximum trip current will be $I_{\text {trip_max }}=I_{\text {phase_pk }}+I_{\text {boost }}=2 I_{\text {phase_pk }}$. A maximum value for the ramp interval $t_{\text {ramp }}$ is then selected that complies with (15), while respecting the $t_{\text {aux_max }}<t_{\text {aux_sw }}$ condition. Hence, (13) becomes:

$$
L_{\mathrm{r}}=\frac{V_{\mathrm{dc}} \cdot t_{\text {ramp_on_max }}}{4 I_{\text {phase_pk }}}
$$

With $t_{\text {res }}$ selected beforehand, the snubber capacitor $C_{\mathrm{r}}$ can be designed with the combination of (11), (9) and (10).

Now that the variable-timing scheme is designed, a fixedtiming scheme follows by demanding that the resulting $t_{\text {res_on_max }}$ interval, the longest that will appear, is equal to the $t_{\text {res }}$ of the variable-timing scheme, while $L_{\mathrm{r}}$ and $C_{\mathrm{r}}$ are kept the same. Additionally, no current or voltage sensing is required, when the trip currents are set equal, that is, $I_{\text {trip }}=I_{\text {boca. }}$ A SABER simulation of the simplified fixed-timing scheme provides a worst-case scenario for the current stress that will be experienced in the inverter. The maximum $I_{\text {off }}$ value defines the repetitive pulsed collector current of the main devices, and the $I_{\text {Lr_rms }}$ value defines the auxiliary device continuous current. Finally, the peak of the tallest $i_{\text {Lr }}$ pulse current defines the pulsed collector current rating of the auxiliary devices.

At this point, the significance of the boost current condition of (15) needs to be discussed. This discussion only applies to variable-timing control, since it is the only scheme that can result in fixed boost currents. The boost current condition ideally guarantees symmetry in the $v_{\text {pole }}$ pulses and an optimised highfrequency response, but comes at the cost of increased current stress and power loss. Firstly, the $i_{\text {Lr }}$ pulses during turn-ons will be too high, since $I_{\text {trip }}=I_{\text {boost }}+I_{\text {phase }}$ will increase from $I_{\text {phase_pk }}$ when $I_{\text {phase }}=0$, to $2 \cdot I_{\text {phase_pk }}$ when $I_{\text {phase }}=I_{\text {phase_pk }}$. Conventionally, $I_{\text {boost }}$ must be just large enough to overcome the impact that the parasitic resistance of the auxiliary circuit has on resonance [43]. In other words, $I_{\text {boost }}$ provides extra energy to the resonant circuit, so that $v_{\text {pole }}$ can swing fully to the opposite DC rail. If the boost current condition is not applied, $I_{\text {boost }}$ can be reduced to a value that is small enough for successful ZVS, and at the same time for decreased current stress and power loss in the inverter.
The turn-on transition at $I_{\text {phase_pk }}$ results in the tallest and widest $i_{\text {Lr }}$ pulse during $T_{1}$. Its amplitude is described in (17):

$$
I_{\text {Lr_pk_max }}=I_{\text {phase } \_ \text {pk }}+\sqrt{\left(\frac{V_{\text {dc }}}{2 Z_{0}}\right)^{2}+I_{\text {boost }}^{2}}
$$

The $I_{\text {Lr_pk_max }}$ peak current is an indicator for the current stress and the power loss experienced in the auxiliary circuit. A larger value of $L_{\mathrm{r}}$ will increase the resonant impedance $Z_{0}$ and will reduce $I_{\text {Lr_pk_max }}$, but it will lead to a larger and bulkier resonant inductor [32]. Also, it is not ensured that $I_{\text {Lr_rms }}$ will decrease, since for a larger $L_{\mathrm{r}}$, the $t_{\text {ramp }}$ intervals will increase according to (13) and (14), and the $i_{\text {Lr }}$ pulses will widen. Alternatively, $I_{\text {boost }}$ can be reduced, though an investigation of (17) shows that $I_{\text {Lr_pk_max }}$ would not decrease dramatically if $I_{\text {boost }}$ became smaller

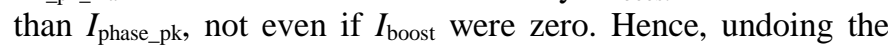
boost current condition just for reducing the value of $I_{\text {boost }}$, would present slight gains in current stress and power loss reduction.

The greatest disadvantage of the boost current condition is not that $I_{\text {boost }}$ becomes too large, but the demand that every turnoff transition must be resonant. This is desirable if every single $v_{\text {pole }}$ pulse is to be sinusoidal and symmetrical in a controllable way. However, the $I_{\text {off }}$ boost current cannot be smaller than $I_{\text {phase_pk, }}$ if every turn-off is to be resonant. For example, the turnoff transition at $I_{\text {phase_pk }}$ can only be resonant if $I_{\text {off }}$ is at least equal to $I_{\text {phase_pk }}$, meaning that $I_{\text {boca }}$ is 0 (since $\left.I_{\text {off }}=I_{\text {phase }}+\left|I_{\text {boca }}\right|\right)$. If, however $I_{\text {off }}<I_{\text {phase_pk }}$, then $\left|I_{\text {boca }}\right|<0$, which is impossible. This way the turn-off transitions around $I_{\text {phase_pk }}$ will not be resonant. Consequently, the only way to examine any drastic trade-offs between power loss/current stress and high-frequency attenuation is to employ capacitive turn-offs that do not require the participation of the auxiliary circuit. As a final note, setting $I_{\text {boost }}=I_{\text {off }}>I_{\text {phase_pk }}$ will not bring about any benefits to either the power loss/current stress, or the high-frequency attenuation.

An ACPI design that focuses primarily on achieving a predictable and attenuated high-frequency response for its output voltage will result in increased power loss and current stress, as opposed to a design that focuses on decreasing switching loss. References [42] and [47] discuss how the boost currents can be selected not only for minimised power loss, but also for other important issues such as DC-link utilization, output voltage quality, and mid-point balancing.

\section{Capacitive Turn-offs}

Fig. 2(c) shows a capacitive switch turn-off, during which $v_{\text {pole }}$ transitions in a linear fashion, rather than a sinusoidal one, since only the snubber capacitors are involved. While the turnon transitions are always resonant, resonant turn-off transitions can be swapped for capacitive ones, but only when $I_{\text {phase }}$ is sufficiently high. Therefore, a threshold $I_{\text {th }}$ is introduced for $I_{\text {phase. }}$. Above $I_{\text {th }}$, the auxiliary circuit is disabled during turn-offs and only the snubber capacitors are used. The control complexity increases this way [42], but current stress and power loss are alleviated, as several $i_{\mathrm{Lr}}$ pulses are dropped. However, the generation of linear $v_{\text {pole }}$ edges is not controllable by the algorithm, but depends on the $I_{\text {phase }}$ level and the value $C_{\mathrm{r}}$ of the snubber capacitors. As $I_{\text {phase }}$ increases, the duration of the linear edges $t_{\text {cap_off }}$ decreases, as in (18).

$$
t_{\text {cap_off }}=\frac{2 C_{\mathrm{r}} V_{\mathrm{dc}}}{I_{\text {phase }}}
$$

$I_{\text {th }}$ should not be set too low, or else $t_{\text {cap_off }}$ will become too long. Here, $C_{\mathrm{r}}$ was designed for the original variable-timing 
scheme and it cannot be altered. As such, $I_{\text {th }}$ is constrained by the modulation scheme. The transition at $I_{\text {phase }}=I_{\text {th }}$ will occur at a specific point in the fundamental cycle. Therefore, the pulsewidth of the SPWM signal at that point in $T_{1}$ must be considered. During the transitions around $I_{\text {th }}$, the longest capacitive turn-off transition of duration $t_{\text {cap_off_max }}$ will be followed by a resonant turn-on transition of duration $t_{\text {res }}$. The pulse-width of the SPWM signal must be large enough to accommodate both transitions. Otherwise, if $t_{\text {cap_off_max }}$ is too long, the $v_{\text {pole }}$ pulse will be deformed or even dropped.

\section{Selection of the Design Parameters}

In this subsection, the design procedure for the experimental 3-phase ACPI prototype is presented. Firstly, a common SPWM modulation scheme is chosen for both the softswitching and the hard-switching inverters with a fundamental frequency of $f_{1}=400 \mathrm{~Hz}$, a switching frequency of $f_{\mathrm{s}}=20 \mathrm{kHz}$, and a modulation index of $m_{\mathrm{A}}=0.83$. The 3-phase load is in a star connection, with $R_{\text {phase }}=10 \Omega$ and $L_{\text {phase }}=2 \mathrm{mH}$. The above circuit conditions lead to a peak phase current of $I_{\text {phase_pk }}=18 \mathrm{~A}$, when the DC-link voltage is $V_{\mathrm{dc}}=500 \mathrm{~V}$.

Since the $v_{\text {pole }}$ frequency response is to be optimised with respect to hard switching, a long enough $t_{\text {res }}$ is demanded, during which the $v_{\text {pole }}$ edges are shaped. Additionally, a symmetrical $v_{\text {pole }}$ pulse-train is desired under variable timing. Under these considerations, the resonant intervals are demanded to be $t_{\text {res_on }}=t_{\text {res_off }}=1.2 \mu \mathrm{s}$. At the same time, the boost current condition of (15) is applied for a symmetrical $v_{\text {pole }}$ pulse-train. Thus, $I_{\text {boost }}=I_{\text {off }}=I_{\text {phase_pk }}=18 \mathrm{~A}$ is set.

The SPWM scheme imposes a constraint of a minimum pulse-width of $d_{\min } \cdot T_{\mathrm{s}}=4.2 \mu \mathrm{s}$. The resultant pulse-width of the widest and tallest $i_{\mathrm{Lr}}$ current pulse is demanded to be quite smaller at $t_{\text {aux_max }}=2 \mu \mathrm{s}$. With $t_{\text {res }}=1.2 \mu \mathrm{s}$, the maximum ramp interval that will appear under the variable-timing scheme is $t_{\text {ramp_on_max }}=400 \mathrm{~ns}$, since $t_{\text {aux_max }}=2 \cdot t_{\text {ramp_on_max }}+t_{\text {res. }}$. The $t_{\text {aux_max }}$ pulse-width can now be accommodated by a fixed auxiliary switch conduction period of $t_{\text {aux_sw }}=2.2 \mu \mathrm{s}$.

Next, the values of the resonant components are estimated. Since $t_{\text {ramp_on_max }}=400 \mathrm{~ns}$ and the boost current condition is set as shown above, (16) results in $L_{\mathrm{r}}=2.7 \mu \mathrm{H}$. Then, since $t_{\text {res }}=1.2 \mu$ s and $I_{\text {boost }}=18 \mathrm{~A},(11)$ leads to $C_{\mathrm{r}}=47 \mathrm{nF}$.

Fixed-timing control is then designed, while maintaining $L_{\mathrm{r}}$ and $C_{\mathrm{r}}$, and demanding that the maximum resonant interval that will appear during $T_{1}$ is $t_{\text {res_on_max }}=1.2 \mu$ s. Moreover, the fixedtiming scheme is simplified by setting the trip currents to a fixed value of $I_{\text {trip }}=I_{\text {boca }}=36 \mathrm{~A}$, which can be achieved with a fixed $t_{\text {ramp }}=400$ ns. Simulating this scheme in SABER predicts that the RMS value of $i_{\mathrm{Lr}}$ is $I_{\mathrm{Lr} \_ \text {rms }}=15 \mathrm{~A}$, and that during the turnon transition at $I_{\text {phase_pk }}$, the largest $i_{\text {Lr }}$ peak is $I_{\text {Lr_pk_max }}=68 \mathrm{~A}$. Furthermore, during the turn-off transition at $I_{\text {phase_pk }}$ the largest boost current for turn-offs is $I_{\text {off_max }}=54 \mathrm{~A}$. These parameters are useful for selecting the device current ratings.

Finally, a phase current threshold of $I_{\text {th }}=12 \mathrm{~A}$ is introduced to the variable-timing scheme, above which the auxiliary circuit is disabled and the turn-off transitions are realised only by $I_{\text {phase }}$ and the snubber capacitors. With $I_{\mathrm{th}}=12 \mathrm{~A}$, the longest capacitive turn-off that will appear during $T_{1}$ is $t_{\text {cap_off_max }}=3.9 \mu \mathrm{s}$, according to (18).

In summary, a fixed $t_{\text {res }}$ value is selected for generating a symmetrical output voltage pulse-train under variable-timing control, and the boost current condition is set. Then, a global fixed auxiliary switch conduction period $t_{\text {aux_sw }}$ is set. Next, the
$L_{\mathrm{r}}$ and $C_{\mathrm{r}}$ are calculated. A fixed-timing scheme is then designed for sizing the main and auxiliary devices. Lastly, capacitive turn-offs are introduced for examining any trade-offs that may arise between the decreased power loss/current stress, and the high-frequency content attenuation.

\section{EXPERIMENTAL RESULTS}

A 3-phase 5-kW ACPI prototype was built for validating the proposed method of high-frequency harmonic attenuation. Fig. 8 shows a single-phase ACPI module. Three of them connected to a common DC link, form the 3-phase ACPI. Two 1200V/40A IKW40N120T2 IGBT copacks from Infineon constitute the main devices $\left(\mathrm{S}_{1}\right.$ and $\left.\mathrm{S}_{4}\right)$, and two 600V/35A NGTB35N60FL2WG IGBT copacks from ON Semiconductor the auxiliary ones $\left(\mathrm{S}_{\mathrm{a} 1}\right.$ and $\left.\mathrm{S}_{\mathrm{a} 4}\right)$. The resonant components can be easily disconnected for hard-switching tests.

A Xilinx XC3S400 FPGA executes the PWM algorithm. Under hard switching it is input with a $t_{\text {dead }}=1.2 \mu$ s dead-time, and with a $t_{\text {aux_sw }}=2.2 \mu$ s under soft switching. Variable timing relies on the online calculation of (13) and (14) by a TI F28335 DSP, which is based on the manually-input boost current condition of $I_{\text {boost }}=I_{\text {off }}=18 \mathrm{~A}$, and the $V_{\mathrm{dc}}$ and $I_{\text {phase }}$ levels. These are sensed by AD7667 analog-to-digital converters. The $I_{\text {phase }}$ polarity selects the appropriate auxiliary switch. In addition, capacitive turn-offs are realised by introducing a threshold of $I_{\text {th }}=12$ A to the variable-timing scheme. Fixed timing requires only the input of $t_{\text {ramp }}=400 \mathrm{~ns}$ that leads to the condition of $I_{\text {trip }}=I_{\text {boca }}=36$ A.

A modified gate driver based on a Schmitt trigger can sense the zero-crossing of the voltage across the main devices [48], for turning on the incoming switch at the end of $t_{\text {res }}$, making for load-adaptive control. This however will add more complexity. Instead, a fixed 1.2- $\mu$ s delay is introduced to the PWM algorithm that starts as soon as the outgoing switch is gated off. After the delay, the incoming switch is gated on. Experimental trial and error is required for tuning this delay.

3-phase experiments are presented with a DC-link voltage of $V_{\mathrm{dc}}=450 \mathrm{~V}$, under hard switching, fixed timing, variable timing, and variable timing with capacitive turn-offs. Only phase A results are presented, due to the 3-phase symmetry.

\section{A. Fundamental Cycle and ZVS}

Fig. 9 shows the phase A current $i_{\mathrm{A}}$, the output voltage $v_{\text {pole }}$, and the inductor current of phase $\mathrm{A} i_{\mathrm{LrA}}$, for two fundamental cycles under fixed timing in Fig. 9(a), variable timing in

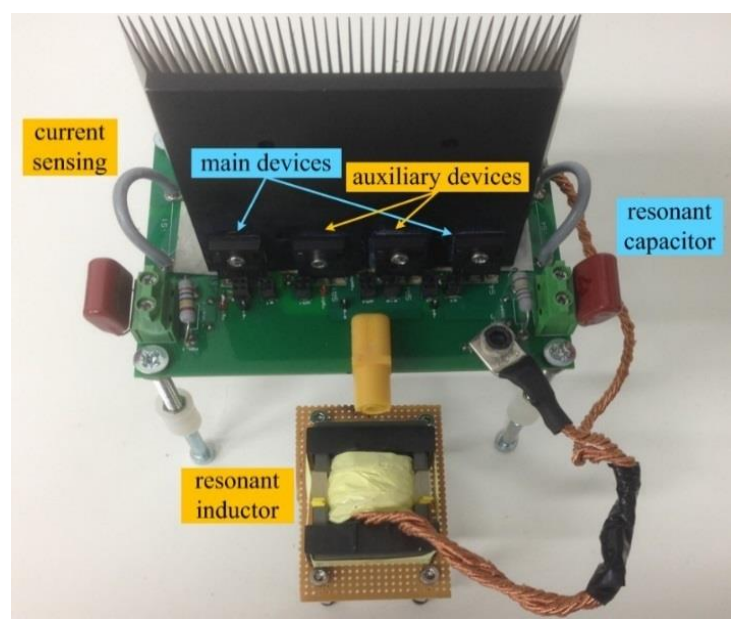

Fig. 8. A single-phase module of the ACPI prototype. 
Fig. 9(b) and variable timing with capacitive turn-offs in Fig. 9(c). In Fig. 9(a), $i_{\text {LrA }}$ does not vary much with $i_{\mathrm{A}}$, as the current pulses are unnecessarily high, whereas in Fig. 9(b) the variation of $i_{\text {LrA }}$ with $i_{\mathrm{A}}$ is stronger, as the pulse amplitudes get smaller in certain intervals. This way, the RMS value is $I_{\text {LrA_rms }}$ $=11.2 \mathrm{~A}$ under fixed timing, and $I_{\text {LrA_rms }}=9.5 \mathrm{~A}$ under variable timing. In Fig. 9(c), the $i_{\mathrm{LrA}}$ turn-off pulses (negative pulses for $i_{\mathrm{A}}>0$, positive pulses for $i_{\mathrm{A}}<0$ ) above the $I_{\mathrm{th}}$ level are dropped. This way, $I_{\text {LrA_rms }}$ is even smaller at $8.9 \mathrm{~A}$. Overall, variabletiming control can decrease current stress and the auxiliary circuit loss, since the $i_{\text {LrA }}$ pulses are built according to the phase current level. Capacitive turn-offs further reduce current stress and power loss.

Fig. 10 shows the ZVS transitions of the main switch $S_{1}$ at peak phase current $I_{\mathrm{A} \_\mathrm{pk}}$. Current $i_{\mathrm{S} 1}$ and voltage $v_{\mathrm{S} 1}$ are shown during the turn-on and turn-off transitions under hard switching in Figs. 10(a) and (b), and under variable timing in Figs. 10(c) and (d). The capacitive turn-off transition is shown in Fig. 10(e). During the transition of Fig. 10(a), the reverse recovery current of the main diode $\mathrm{D}_{4}$ causes $i_{\mathrm{S} 1}$ to peak to double the $i_{\mathrm{A}}$ level. Also, the tail of $i_{\mathrm{S} 1}$ in Fig. 10(b) worsens the hard-switching turn-off loss. In contrast, soft switching decouples the edges and minimize switching loss, as shown in Figs. 10(c), (d) and (e). Variable timing fully decouples the edges in Fig. 10(c). There is a large overshoot in $i_{\mathrm{S} 1}$ though, due to the ringing caused by the parasitic inductance of the switching loop. In Fig. 10(d), $i_{\mathrm{S} 1}$ peaks to 29.4 A due to the $I_{\text {off }}$ component. Also, the edges in Fig. 10(d) are not decoupled fully due to the tail current bump of $6.6 \mathrm{~A}$ that $i_{\mathrm{S} 1}$ exhibits. This peculiar bump occurs in IGBTs

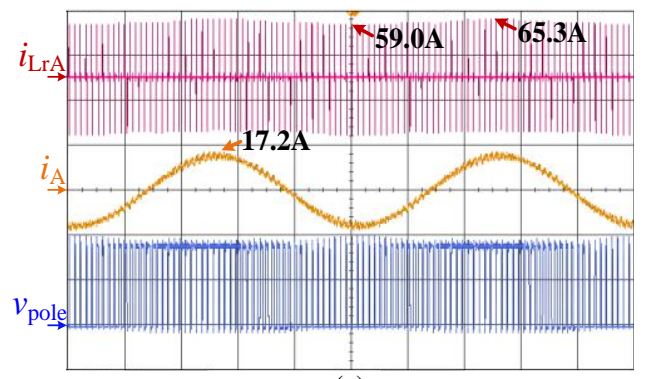

(a)

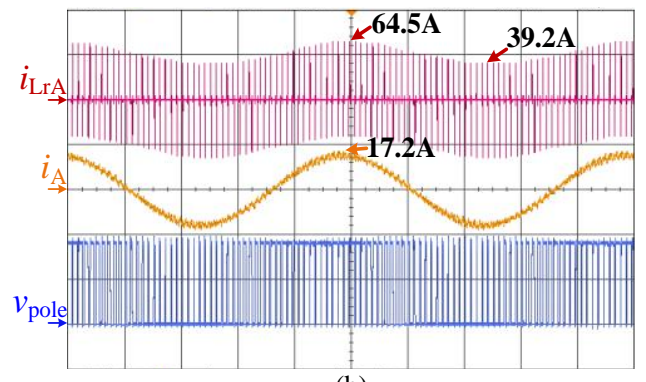

(b)

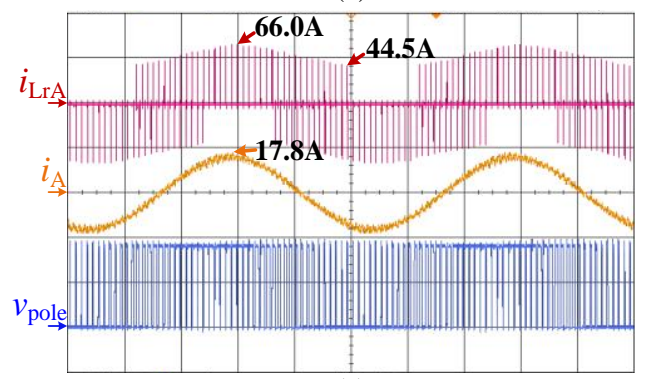

(c)

Fig. 9. Phase A current $i_{\mathrm{A}}$, output voltage $v_{\text {pole }}$, and phase A inductor current $i_{\text {LrA }}$ under (a) fixed timing, (b) variable timing, and (c) variable timing with capacitive turn-offs over two fundamental cycles. $i_{\mathrm{A}} 20 \mathrm{~A} / \mathrm{div}, v_{\text {pole }} 250 \mathrm{~V} / \mathrm{div}$, $i_{\text {LrA }} 50 \mathrm{~A} / \mathrm{div}$, time $0.5 \mathrm{~ms} / \mathrm{div}$. only when they turn off under ZVS [49]. Generally, more turnoff loss is incurred in the ACPI than turn-on loss [33], unless capacitive turn-offs are used, as in Fig. 10(e), in which case the ACPI operates as an almost lossless turn-off snubber. Overall, soft-switching decouples the transitioning edges and achieves ZVS, especially under resonant turn-ons and capacitive turnoffs, whereas hard switching does not, with the penalty of increased switching loss.

\section{B. Resonant Transitions \& Output Voltage Edge Generation}

Of major importance in this work is how the four switching modes generate the output voltage edges. This comparison is

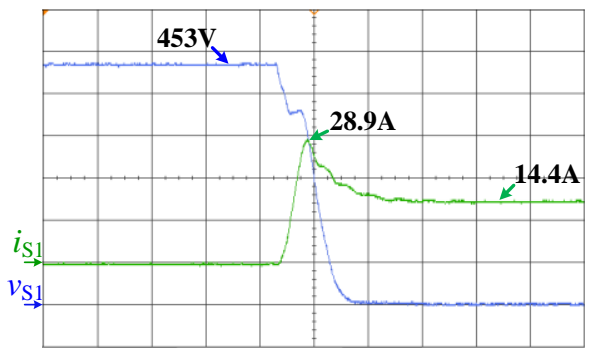

(a)

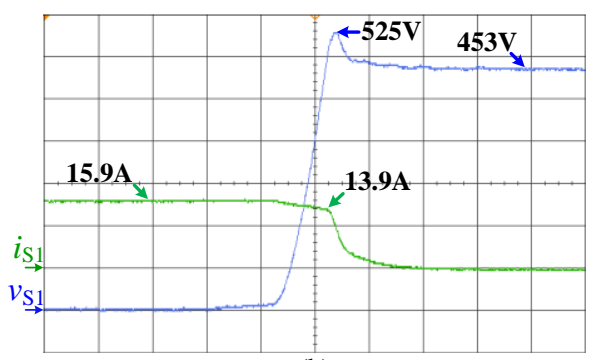

(b)

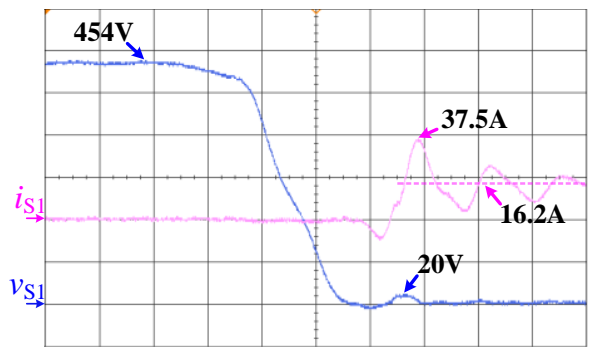

(c)

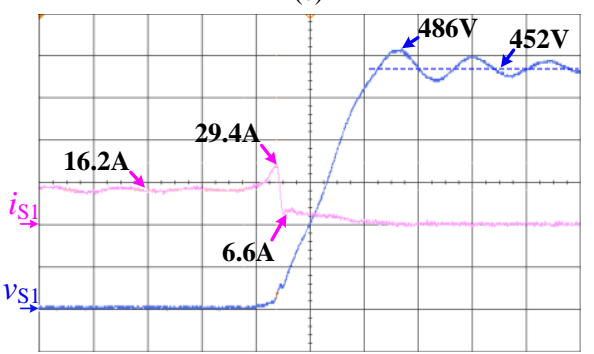

(d)

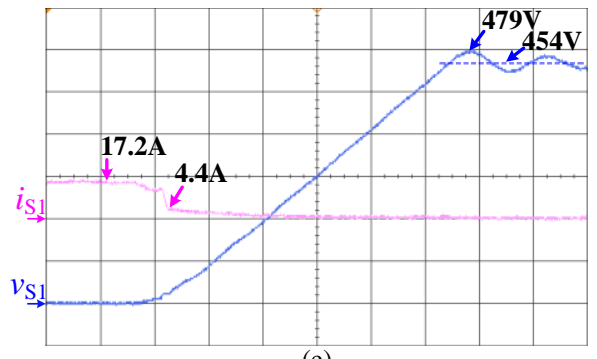

(e)

Fig. 10. Switching current $i_{\mathrm{S} 1}$ and voltage $v_{\mathrm{S} 1}$ during the transitions of $\mathrm{S}_{1}$ at peak phase current $I_{\text {A pk }}$. (a) Hard-switched turn-on and (b) turn-off, (c) variabletiming turn-on and (d) turn-off, and (e) variable timing with capacitive turn-off. Hard switching: $v_{\mathrm{S} 1} 80 \mathrm{~V} / \mathrm{div}, i_{\mathrm{S} 1} 10 \mathrm{~A} / \mathrm{div}$, time $200 \mathrm{~ns} / \mathrm{div}$. Soft switching: $v_{\mathrm{S} 1}$ $80 \mathrm{~V} / \mathrm{div}, i_{\mathrm{S} 1} 20 \mathrm{~A} / \mathrm{div}$, time $500 \mathrm{~ns} / \mathrm{div}$. 
shown in Fig. 11, where $S_{1}$ is shown to first turn off and then to turn on, at $I_{\text {A_pk }}$. The hard-switched edges in Fig. 11(a) transition quickly and linearly in a matter of $200 \mathrm{~ns}$. In contrast, the softswitched edges in Figs. 11(b), (c) and (d) are slower and smoother, with their duration spanning a range of $800 \mathrm{~ns}$ to $3 \mu \mathrm{s}$. However, the resonant soft-switched edges are not shaped in an ideal sinusoidal manner, nor are their corners completely smooth. In Fig. 11(b), fixed timing generates edges that are unequal in duration by $400 \mathrm{~ns}$. As discussed in Sections IV and $\mathrm{V}$, since the trip currents are fixed under fixed-timing control, the resonant energy is provided in an erratic way, and not according to the phase current level. As a result, the two $i_{\text {LrA }}$ pulses are close in amplitude, even if the turn-off pulse need not be that large at $I_{\mathrm{A} \_p k}$. In contrast, variable timing in Fig. 11(c), generates edges of almost equal duration, as it provides the resonant energy according to the $i_{\mathrm{A}}$ level. Thus, the turn-off pulse drops by $20 \mathrm{~A}$. When capacitive turn-offs are introduced in Fig. 11(d), the turn-off edge becomes linear and almost 2.5
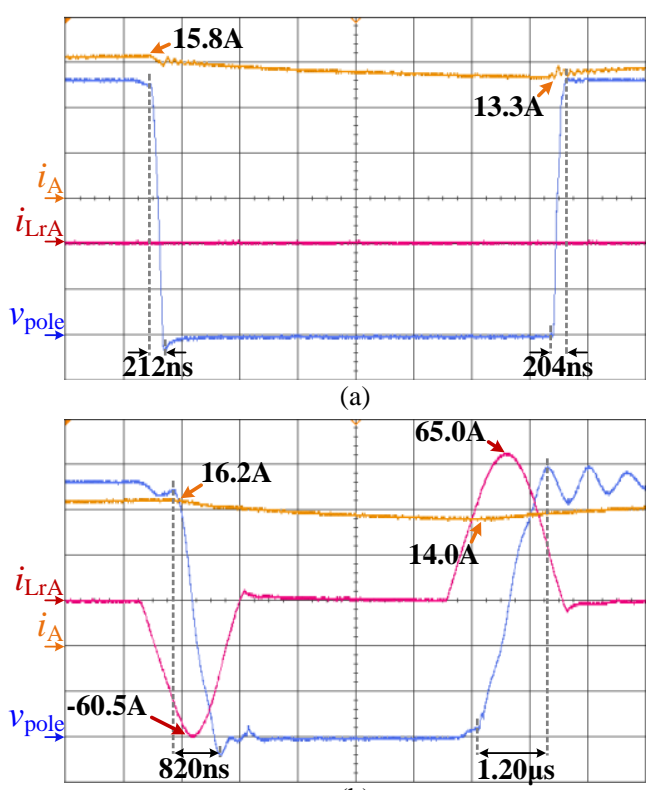

(b)

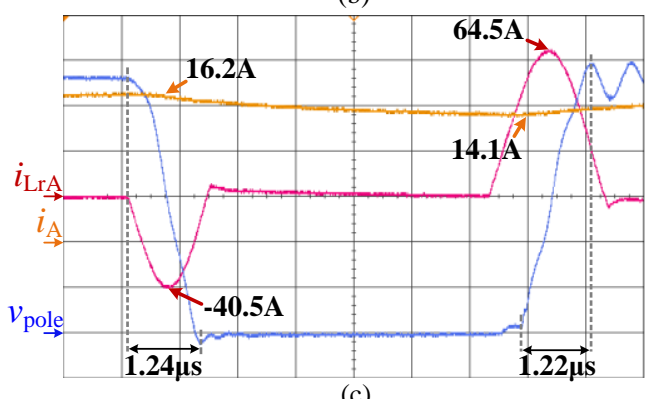

(c)

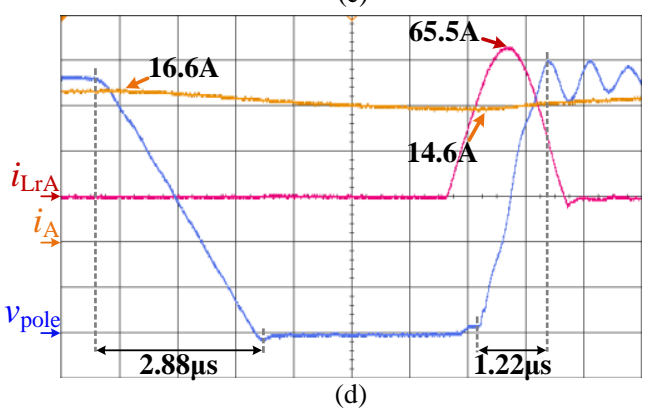

Fig. 11. Phase current $i_{\mathrm{A}}$, output voltage $v_{\text {pole }}$, and inductor current $i_{\text {LrA }}$ during the transitions of $S_{1}$ at $I_{\mathrm{A}_{-} \mathrm{pk}}$ under (a) hard switching, (b) fixed timing, (c) variable timing, and (d) variable timing with capacitive turn-offs. $i_{\mathrm{A}} 5 \mathrm{~A} / \mathrm{div}$, $v_{\text {pole }} 80 \mathrm{~V} / \mathrm{div}, i_{\mathrm{LrA}} 20 \mathrm{~A} / \mathrm{div}$, time $1 \mu \mathrm{s} /$ div . times longer than the turn-on edge, since the snubber capacitors are quite large at $47 \mathrm{nF}$.

All the linear $v_{\text {pole }}$ edges differ in duration as they depend on the $i_{\mathrm{A}}$ level. They are not dictated by the control algorithm, as is the intention with the resonant edges. In addition, it is seen that these $v_{\text {pole }}$ pulses that appear whenever the $I_{\text {th }}$ threshold is exceeded, have a resonant and a linear edge. This introduces asymmetry in their duration and shape. The uncontrollability and asymmetry caused by the capacitive turn-offs can lead to an unpredictable high-frequency response, with the linearly-shaped edges potentially contributing to a higher harmonic content.

The resonant edges are not completely sinusoidal either. Fig. 12 examines the turn-on transition of $S_{1}$ at $I_{\mathrm{A} \_p k}$ and under variable timing, where secondary effects take place before, during, and after resonance. As $i_{S 4}$ through the outgoing $\mathrm{D}_{4}$ ramps up along with $i_{\text {LrA }}$ during $t_{1}$, a di/dt-induced voltage drop across the negative rail parasitic inductance appears as a 16-V undershoot on $v_{\text {pole. }}$. As $i_{\text {LrA }}$ keeps ramping up, $i_{\mathrm{S} 4}$ starts flowing through $\mathrm{S}_{4}$ until it reaches $30.6 \mathrm{~A}$. Then in a matter of $80 \mathrm{~ns}$ (interval $t_{2}$ ), $i_{\mathrm{S} 4}$ abruptly drops to near zero, since the turn-off delay has transpired and the IGBT starts turning off [14]. At the same time, the $i_{\mathrm{Cr} 4}$ capacitor current jumps to $28.5 \mathrm{~A}$, as it takes up the $I_{\text {boost }}$ current from $\mathrm{S}_{4}$ to commence resonance. This swift change in $i_{\mathrm{Cr} 4}$ forces $v_{\text {pole }}$ to jump to $42 \mathrm{~V}$, with a high $\mathrm{d} v / \mathrm{dt}$, at the end of $t_{2}$.

The true $I_{\text {trip }}$ value of $i_{\text {LrA }}$ is 50.5 A for this turn-on transition. Ideally at $I_{\mathrm{A} \_\mathrm{pk}}$, it should be near $36 \mathrm{~A}$. The major reason for this discrepancy is the turn-off delay of $S_{4}$ that forces $i_{\mathrm{LrA}}$ to keep ramping up beyond the prescribed $I_{\text {trip }}$. Another reason is that the

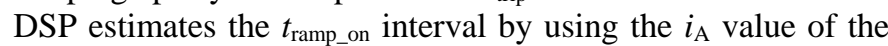
previous switching cycle, due to the delay introduced by the AD7667 converters. This way, $S_{4}$ is gated off with a delay estimated for one $i_{\mathrm{A}}$ value, while resonance is happening under another $i_{\mathrm{A}}$ value. Thus, the $I_{\text {boost }}$ value is altered, and the $v_{\text {pole }}$ edges stray from their ideal duration and shape. This makes for a more unpredictable frequency response and for a harder estimation of the spectral envelope.

During the resonance of $t_{3}$, the parasitic inductance of the negative rail and the output node is in the path of $i_{\mathrm{Cr} 4}$ and influences its oscillation. As such, $i_{\mathrm{Cr} 4}$ does not look similar to $i_{\mathrm{Lr}}$ but oscillates with a frequency of $f_{\mathrm{a}}=1.9 \mathrm{MHz}$. This causes undulations on the $v_{\text {pole }}$ edge and alters its sinusoidal shape. After $t_{5}, i_{\mathrm{Cr} 4}$ oscillates at $f_{\mathrm{b}}=1.4 \mathrm{MHz}$, signifying that there is no $L_{\mathrm{r}}$ contribution anymore. This problem is specific to this prototype and is exacerbated by the presence of the snubber capacitors. Hard switching does not exhibit these oscillations. A better-engineered PCB could mitigate this issue.

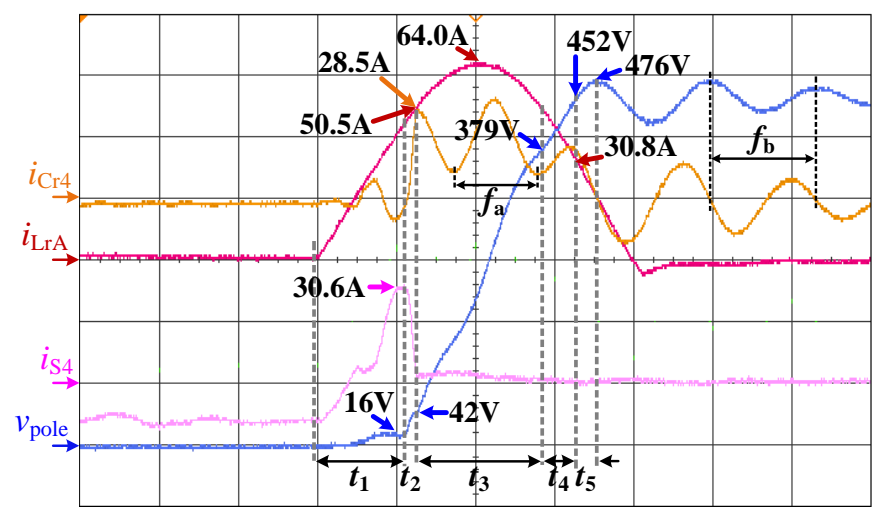

Fig. 12. Capacitor current $i_{\mathrm{Cr} 4}$, output voltage $v_{\text {pole }}$, inductor current $i_{\mathrm{LrA}}$, and switching current $i_{\mathrm{S} 4}$ during the turn-on transition of $\mathrm{S}_{1}$ at $I_{\mathrm{A} \_ \text {pk }}$ under variable timing. $i_{\mathrm{Cr} 4} 20 \mathrm{~A} / \mathrm{div}, v_{\text {pole }} 80 \mathrm{~V} / \mathrm{div}, i_{\mathrm{LrA}} 20 \mathrm{~A} / \mathrm{div}, i_{\mathrm{S} 4} 20 \mathrm{~A} / \mathrm{div}$, time $500 \mathrm{~ns} / \mathrm{div}$. 
The $770 \mathrm{~ns}$ of $t_{3}$ are the symmetrical portion of resonance, since $i_{\mathrm{LrA}}$ is bounded by $I_{\text {trip }}=50.5 \mathrm{~A}$. However, $v_{\text {pole }}$ does not reach the DC rail until $250 \mathrm{~ns}$ later, at the end of $t_{4}$, when $i_{\mathrm{LrA}}=$ $30.8 \mathrm{~A}$. This is $20 \mathrm{~A}$ below $I_{\text {trip. Therefore, full resonance lasts }}$ for $t_{\text {res }}=t_{3}+t_{4}=1020 \mathrm{~ns}$ and it is asymmetrical. This is due to the resonant circuit's parasitic resistance [31]. If a small $R$ is considered in series with $L_{\mathrm{r}}$ in the LC circuits of Figs. 5(a) and (b), the resonant magnitudes will assume an underdamped waveform of the form $\mathrm{e}^{-x} \sin (x)$. This $R$ seems to be increased, and the reason can be traced in the inductor winding. The $i_{\mathrm{LrA}}$ waveform is expected to be rich in high-frequency harmonics, exacerbating the skin and proximity effects in the winding and increasing its $\mathrm{AC}$ resistance. Regardless, $i_{\mathrm{LrA}}$ keeps resonating with $v_{\text {pole }}$ until the DC rail is reached at the end of $t_{4}$, and ZVS is achieved, with no jump in the $\mathrm{d} v / \mathrm{dt}$ and no current spike in the incoming switch. Finally, it seems that the parasitic resistance counters the negative effect of the turn-off delay. The turn-off delay pushes resonance to transpire faster by increasing the value of $I_{\text {trip }}$, but the parasitic resistance slows it down.

$130 \mathrm{~ns}$ later at the end of $t_{5}, v_{\text {pole }}$ overshoots slightly to $476 \mathrm{~V}$ and starts oscillating at $f_{\mathrm{b}}=1.4 \mathrm{MHz}$, along with $i_{\mathrm{S} 4}$. This overshoot and the subsequent oscillation are not considered part of the $v_{\text {pole }}$ edge, as originally assumed in Figs. 11(b), (c), and (d). Rather, these phenomena are translated as increased harmonic content around the frequency of the ringing [3]. The $16-\mathrm{V}$ undershoot during $t_{1}$ and the sudden jump during $t_{2}$ will similarly have their own contributions to the $v_{\text {pole }}$ spectrum. As such, the $v_{\text {pole }}$ edge actually lasts for $t_{2}+t_{3}+t_{4}=1100 \mathrm{~ns}$, with resonance shaping it for $1020 \mathrm{~ns}$.

\section{Effect of the Main Switch Gate Resistor on Resonance}

Since the turn-off delay of the outgoing switch has such a strong influence on resonance, the effect of the main switch gate resistor $R_{\mathrm{g} \_m a i n}$ is investigated. Fig. 13 shows the variable-timing turn-on transition of $\mathrm{S}_{1}$ at $I_{\mathrm{A} \_\mathrm{pk}}$, for two $R_{\mathrm{g} \_ \text {main }}$ values. Similar results are observed for the turn-off transition, as well.

In Fig. 13(a), $R_{\mathrm{g}_{-} \text {main }}$ is $22 \Omega$. This value is chosen for minimizing any ringing exhibited under hard switching. The turn-off delay between the fall of the $S_{4}$ gate-emitter voltage $v_{\text {ge } 4}$ and the fall of $i_{\mathrm{S} 4}$ is $400 \mathrm{~ns}$. After the $400 \mathrm{~ns}$, the values of $27.8 \mathrm{~A}$ and $49.5 \mathrm{~A}$ are assumed by $i_{\mathrm{S} 4}$ and $i_{\mathrm{LrA}}$, respectively, whereas they should ideally be $18 \mathrm{~A}$ and $36 \mathrm{~A}$. In Fig. 13(b), $R_{\mathrm{g} \_ \text {main }}$ is halved to $11 \Omega$, and overall the transition progresses in a slower, smoother manner with dampened ringing. This is counterintuitive. A lower $R_{\mathrm{g} \_ \text {main }}$ worsens ringing and accelerates the transitions, under hard switching.

The ACPI transitions however, are resonant. By reducing $R_{\mathrm{g} \_m a i n}$, the turn-off delay is now $240 \mathrm{~ns}, i_{\text {LrA }}$ ramps up to a lower $I_{\text {trip }}=37.2 \mathrm{~A}$, and $i_{\mathrm{S} 4}$ peaks at $17 \mathrm{~A}$. The energy put into resonance decreases, forcing the resonant magnitudes to swing to lower amplitudes, with $i_{\text {LrA }}$ peaking at 58.2 A instead of $64 \mathrm{~A}$. Also, the switching loop parasitic inductance is not energized as much, and the ringing in $v_{\text {pole }}$ is suppressed. This way its edge is almost sinusoidal during resonance, and swings seamlessly from the negative to the positive rail with no undershoots or overshoots, in $1580 \mathrm{~ns}$. Lastly, a smaller $R_{\mathrm{g} \_ \text {main }}$ reduces the overall current stress and power loss. With $R_{\mathrm{g} \_ \text {main }}=11 \Omega, I_{\text {LrA_rms }}$ decreases from $9.5 \mathrm{~A}$ to $8.4 \mathrm{~A}$. Overall, a reduced main gate resistor puts less energy into the resonant circuit, resulting in a slower, nearly-sinusoidal edge, with smoother corners, and dampened ringing. All these are beneficial for an attenuated high-frequency response.
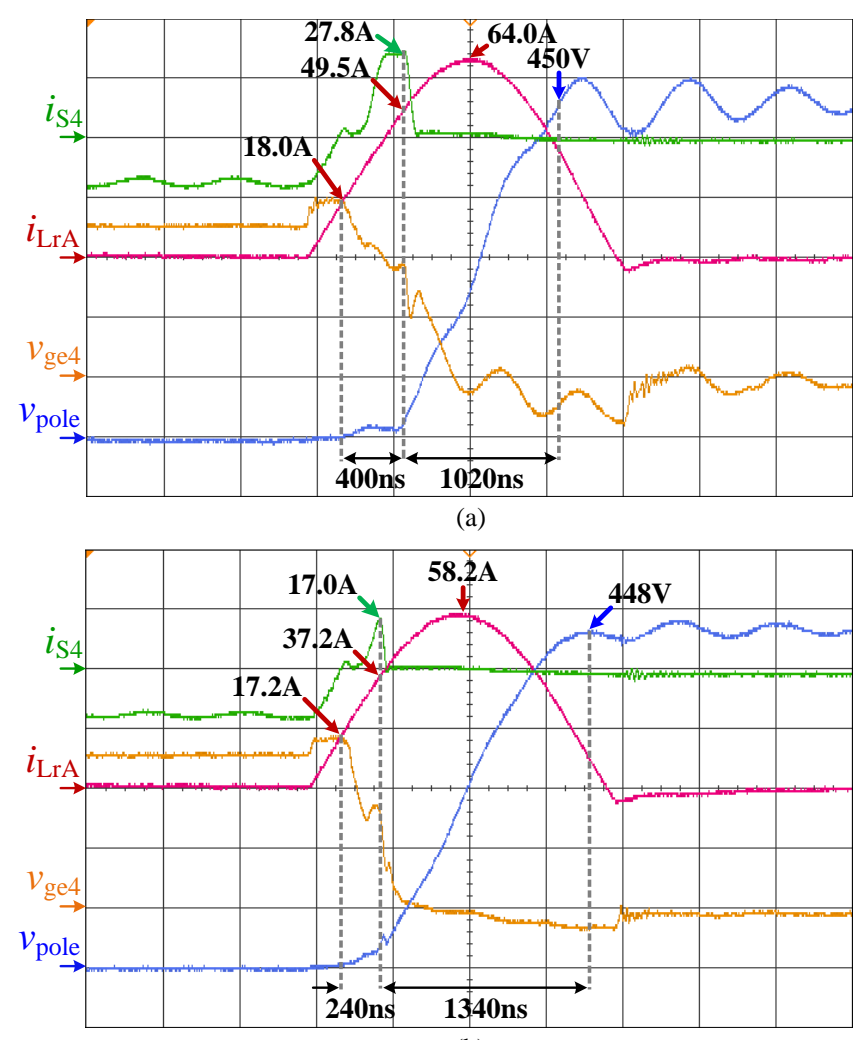

(b)

Fig. 13. Gate-emitter voltage of main device $\mathrm{S}_{4} v_{\text {get }}$, switching current $i_{\mathrm{S} 4}$, output voltage $v_{\text {pole, }}$, and inductor current $i_{\mathrm{LrA}}$ for (a) $R_{\mathrm{g}_{\mathrm{B}} \text { main }}=22 \Omega$, and (b) $R_{\mathrm{g} \_ \text {main }}=11 \Omega$, during the turn-on transition of $\mathrm{S}_{1}$ at $I_{\mathrm{A} \_\mathrm{pk}}$, under variable timing. $v_{\text {ge } 4} 5 \mathrm{~V} / \mathrm{div}, i_{\mathrm{S} 4} 20 \mathrm{~A} / \mathrm{div}, v_{\text {pole }} 80 \mathrm{~V} / \mathrm{div}, i_{\mathrm{LrA}} 20 \mathrm{~A} / \mathrm{div}$, time $500 \mathrm{~ns} / \mathrm{div}$.

Fig. 13 suggests that the variable-timing scheme is not implemented perfectly. In both cases, $i_{\text {LrA }}$ is near $18 \mathrm{~A}$ when $v_{\text {ge } 4}$ starts falling. This is half the intended $I_{\text {trip }}$ value, which might not be enough for $v_{\text {pole }}$ to completely swing to the opposite rail. This can be attributed to the delays associated with sensing the phase current and the DC-link voltage, translating that information into the appropriate $t_{\text {ramp }}$ interval, and then applying the signals to the switches through the gate circuitry that also introduces its own delay. It seems that any outgoing switch turn-off delay is beneficial because it allows $i_{\text {LrA }}$ to keep ramping up and inject more energy into resonance, despite the oscillations, and the fast, misshapen edges.

Lastly in Fig. 13(b), it is noted that even if $I_{\text {trip }}$ is near the prescribed $36 \mathrm{~A}$, the $v_{\text {pole }}$ edge is longer than anticipated. Examining the 1340-ns interval of the edge, it is shaped by the symmetrical part of resonance for approximately $1 \mu \mathrm{s}$, which is closer to what the variable-timing design intended to do in the first place, i.e., to generate a resonant interval of $t_{\text {res }}=1.2 \mu \mathrm{s}$. The rest of the edge is shaped by the asymmetrical part of resonance. With less energy injected into the resonant circuit, the damping due to the parasitic resistance is more pronounced. This further slows down the edge and makes resonance more asymmetrical than in Fig. 13(a). However, ZVS is still successful despite the increased damping.

\section{Efficiency}

The comparison of the four switching modes has yielded substantial differences in $v_{\text {pole }}$ edge generation and ZVS. Also of interest is the efficiency exhibited by each mode. The input and output power of the inverter has been measured by Norma 4000 power analyzers connected at the DC input and the 3-phase output. The efficiency of each switching mode has been 
calculated based on these measurements, at intervals of $500 \mathrm{~W}$. In addition to the default value of $22 \Omega$, the revised value of $R_{\mathrm{g} \_ \text {main }}=11 \Omega$ is also used, and the impact of the capacitive turnoffs is also considered. The first observation is that almost any soft-switching scheme fares worse than hard switching when it comes to power loss. From $3.5 \mathrm{~kW}$ onwards, only variable timing with capacitive turn-offs and $R_{\mathrm{g} \_ \text {main }}=11 \Omega$ is as efficient a scheme as hard switching. At $4.5 \mathrm{~kW}, \eta$ is near $97.6 \%$ under these two schemes.

Fig. 14 shows the efficiency $\eta$ against the DC input power $P_{\text {in. }}$. The first observation is that soft switching is less efficient than hard switching. From $3.5 \mathrm{~kW}$ onwards, only variable timing with capacitive turn-offs and $R_{\mathrm{g} \_ \text {main }}=11 \Omega$ is as efficient as hard switching. They both exhibit $\eta=97.6 \%$, at $4.5 \mathrm{~kW}$.

Soft switching can mitigate the switching loss of the main devices due to the decoupling of the switching magnitudes, but it incurs extra loss, mainly because of the auxiliary circuit. A hard-switching inverter does not exhibit this kind of loss. The auxiliary devices have increased conduction loss due to the $I_{\text {LrA_rms }}$ value that is excessive under some schemes, especially fixed timing. The $i_{\text {Lr }}$ pulses occur this way because the main purpose of this work is high-frequency content attenuation that can be predetermined within the confines of implementation, and not power loss reduction. Also, the resonant inductor is expected to exhibit increased AC resistance and winding loss. Moreover, the fringing effect around the large airgap of the inductor will cause eddy currents in the winding and contribute to its loss. Thus, an enhanced inductor design for coping with the uniqueness of the $i_{\mathrm{Lr}}$ waveform, and for reducing the loss should be considered [50].

The main switches also exhibit increased conduction loss, especially resonant turn-offs, due to the $I_{\text {off }}$ component imposed on the main switching currents. Since the soft-switching schemes have been designed for high-frequency attenuation, $I_{\mathrm{off}}$ is comparable or even larger than the reverse recovery current observed under hard switching, as seen in Figs. 10(a) and (d). This is especially true for the simplified fixed-timing scheme with $I_{\text {trip }}=I_{\text {boca }}$, where $I_{\text {boca }}$ is too high. For example, when the turn-off transition at $I_{\mathrm{A} \_ \text {pk }}$ happens, $I_{\text {off }}=54 \mathrm{~A}$, which is three times the $I_{\text {A_pk }}$ level.

The fact remains that the switching loss of the main devices is still worse under hard-switching, as attested by Figs. 10(a) and

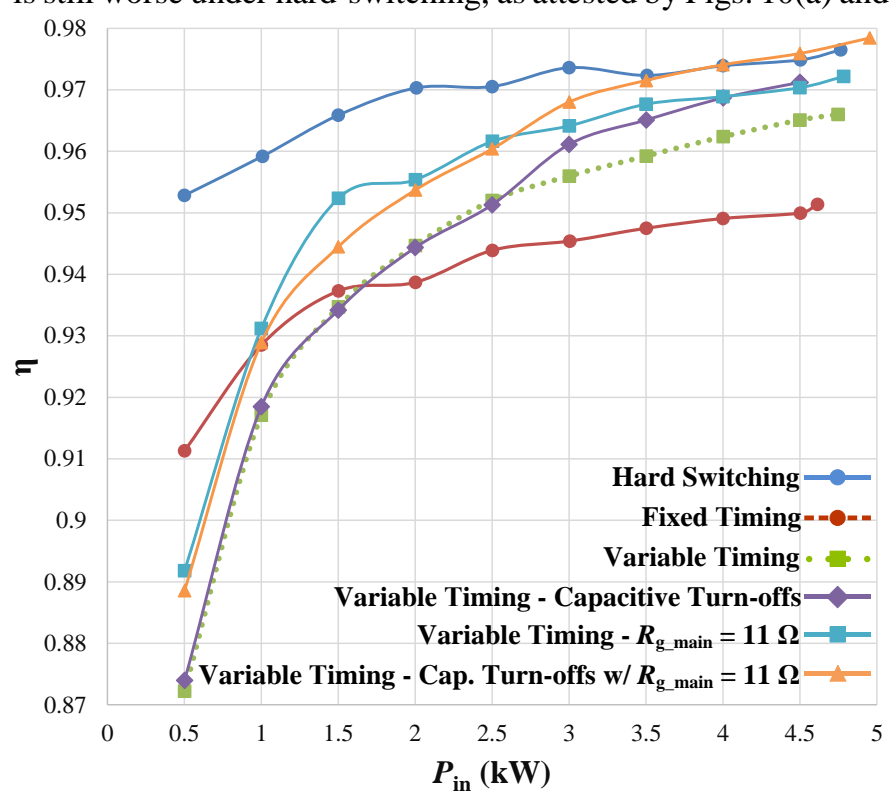

Fig. 14. Power efficiency $\eta$ of the 3-phase ACPI against DC input power $P_{\text {in }}$, under all switching modes. (b). It is important to note though that the decoupling during the resonant turn-offs, like the one shown in Fig. 10(d), is not entirely realised due to the tail-current bump exhibited by IGBTs, when they are switched under ZVS [49].

Variable timing generates smaller $i_{\text {LrA }}$ pulses with decreased $I_{\text {LrA_rms }}$ values and $I_{\text {off }}$ components. This way, it is more efficient than fixed timing. Also, reducing $R_{\mathrm{g} \_ \text {main }}$ leads to less energy being put into any given resonant transition. This is a very simple way of reducing current stress and power loss. The most optimized scheme is variable timing that employs capacitive turn-offs, especially for reduced $R_{\mathrm{g} \_ \text {main. }}$ Not only are several $i_{\mathrm{LrA}}$ pulses dropped, but the decoupling during the capacitive turnoffs is enhanced with regards to a resonant turn-off, as seen in Fig. 10(e).

An ACPI design for purely reducing switching loss without introducing much loss anywhere else should exhibit higher efficiency than hard switching, especially at higher switching frequencies. A resonant inductor that is tailored to the demanding $i_{\text {Lr }}$ waveform [50] can contribute to this. Capacitive turn-offs should also be employed with a $I_{\text {th }}$ that is reasonably low, so that plenty $i_{\text {Lr }}$ pulses are dropped. The remaining pulses should only be as large as necessary for achieving ZVS, without causing too much loss and current stress. Lastly, a reasonably small $R_{\mathrm{g} \_ \text {main }}$ should be employed.

\section{E. Output Voltage Frequency Response}

Fig. 15 shows the frequency response of $v_{\text {pole }}$, under hard switching, fixed timing and variable timing for a main gate resistor of $R_{\mathrm{g} \_ \text {main }}=22 \Omega$, and the variable timing spectrum for $R_{\mathrm{g} \_ \text {main }}=11 \Omega$. 20 fundamental cycles of the time-domain waveforms are captured at $V_{\mathrm{dc}}=450 \mathrm{~V}$ with a Rohde $\&$ Schwarz RTO1024 oscilloscope. A resolution of $5 \mathrm{~ns}$ and a sample rate of $200 \mathrm{MSa} / \mathrm{s}$ give a record length of $10 \mathrm{MSa}$ for each waveform. The noise floor at high frequencies is decreased by averaging the waveforms 5000 times. Then, Matlab performs an optimized Fast Fourier Transform on the time-domain data for obtaining the frequency-domain results.

The most important observation is that from near $600 \mathrm{kHz}$ onwards, all soft-switching schemes exhibit less harmonic content than hard switching because of the longer and smoother $v_{\text {pole }}$ edges. The PWM region below $600 \mathrm{kHz}$ has an envelope slope of nearly $-20 \mathrm{~dB} / \mathrm{dec}$ that is common to all switching modes. However, the $-40 \mathrm{~dB} / \mathrm{dec}$ slope appears earlier under soft switching than hard switching. Moreover, a $-60 \mathrm{~dB} / \mathrm{dec}$

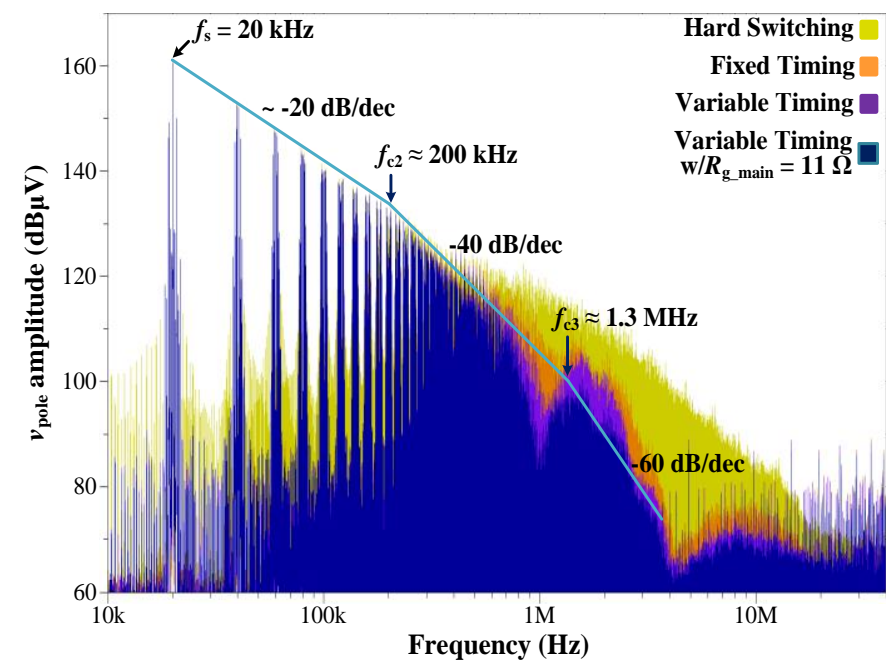

Fig. 15. Experimental output voltage frequency response under hard switching, fixed timing, variable timing, and variable timing with $R_{\mathrm{g} \_ \text {main }}=11 \Omega$. 
slope appears under soft switching, which further contributes to the high-frequency content attenuation. The existence of both the -40 and $-60 \mathrm{~dB} / \mathrm{dec}$ slopes means that the $v_{\text {pole }}$ edges behave more like $\mathrm{S}$-shaped ones, rather than sinusoidal ones that only exhibit the $-60 \mathrm{~dB} / \mathrm{dec}$ slope. This is a consequence of the ACPI's quasi-resonant action, which takes part of the free oscillation of the resonant circuit and applies it to the resonant magnitudes, as observed in Fig. 5(c). Hence, most of the middle stretch of the edges has an almost fixed $\mathrm{d} v / \mathrm{dt}$ rate that makes them S-shaped. Conversely, as expected, the hard-switched $v_{\text {pole }}$ pulse-train behaves like a trapezoidal one, with only the $-40 \mathrm{~dB} / \mathrm{dec}$ slope observable up to $40 \mathrm{MHz}$, before the noise floor is reached.

The frequency-domain results of the $v_{\text {pole }}$ pulse-train with linear edges due to capacitive turn-offs are not shown here, because they are almost identical to the ones of their respective variable-timing scheme without the capacitive turn-offs. This means that the number of the linear edges is not large enough to convincingly influence the spectrum. Additionally, the linear edges are so long that they do not exacerbate the high-frequency content because of their shape. It is seen that in this work, capacitive turn-offs are useful for reducing current stress and power loss in the inverter without altering the high-frequency attenuation capability of the variable-timing scheme, albeit at the cost of increased control complexity.

Of all the schemes presented in Fig. 15, variable timing with a reduced $R_{\mathrm{g} \_ \text {main }}$ gate resistor of $11 \Omega$ has the best performance, since it generates the longest and smoothest edges in the time domain, as seen in Fig. 13(b). With this simple alteration, gains in both the power efficiency and the high-frequency harmonic attenuation are achieved as attested by Figs. 14 and 15, respectively. This soft-switching scheme also has the most predictable frequency response, because the edges are shaped with no overshoots and undershoots, and because the ringing both during the transition and at steady state is suppressed. This is not the case with the default $R_{\mathrm{g} \_ \text {main }}=22 \Omega$. The favourable edge shape when $R_{\mathrm{g} \_ \text {main }}=11 \Omega$ allows for the approximation of the spectral envelope and its corner frequencies, as shown in Fig. 15. It is assumed that every single edge of this pulse-train is the same as the one shown in Fig. 13(b), with a rise time of $t_{\mathrm{r}}=1580 \mathrm{~ns}$ and a first-derivative rise time of $t_{\mathrm{r}(\mathrm{dv} / \mathrm{dt})}=240 \mathrm{~ns}$. With these parameters, the second corner frequency $f_{\mathrm{c} 2}$ should be $237.5 \mathrm{kHz}$, according to (3). This is close to the observed $f_{\mathrm{c} 2}=200 \mathrm{kHz}$ in Fig. 15 . The third corner frequency $f_{\mathrm{c} 3}$ should theoretically be $1.33 \mathrm{MHz}$, according to (4), a value that is very close to the observed $1.3 \mathrm{MHz}$.

Peaks of exacerbated harmonic content are seen between 1 and $3 \mathrm{MHz}$ in the three soft-switching spectra, which interrupt the $-60 \mathrm{~dB} / \mathrm{dec}$ slope. As seen in Figs. 11(b), 11(c), 12 and 13, these peaks are due to the ringing observed in the time domain, under the soft-switching schemes. The first peak is concentrated around a frequency near $1.4 \mathrm{MHz}$, and the second one, seen merging with the first, is centred on $2 \mathrm{MHz}$. The first peak is attributed to the 1.4- $\mathrm{MHz}$ ringing seen on the $v_{\text {pole }}$ waveform as soon as it reaches steady state, while the second peak is due to the $1.9-\mathrm{MHz}$ ringing of the capacitor current $i_{\mathrm{Cr} 4}$ that is responsible for the undulation on the $v_{\text {pole }}$ edges. As observed in Fig. 13(b), the ringing is attenuated when $R_{\mathrm{g} \_ \text {main }}=11 \Omega$, and this is reflected in the corresponding spectrum. It is repeated here that these oscillations are inherent to the manufactured PCB used in this work. A better engineered PCB can mitigate these phenomena.
Nevertheless, the variable-timing scheme with $R_{\mathrm{g} \_ \text {main }}=11 \Omega$ results in a maximum harmonic content attenuation of $37 \mathrm{~dB}$ at $4 \mathrm{MHz}$, with regards to hard switching.

\section{VIII.CONCLUSIONS}

This paper has presented a method for designing ACPI converters mainly for attenuating high frequency EMI purposes. It has been shown that the generation of symmetrical $v_{\text {pole }}$ pulses is made possible with variable-timing control, which allows for a frequency response with more predictable and predetermined spectral envelopes. Capacitive turn-offs, though adding to the control complexity, were shown to alleviate current stress and power loss even further, without altering the high-frequency response, since the linear $v_{\text {pole }}$ edges they produced were long and low in number. Lastly, it was shown that reducing the main gate resistor value is a simple and effective way of reducing high-frequency harmonic content, power loss, and current stress under any soft-switching scheme. Thus, a 3-phase, 5-kW ACPI controlled under a variable-timing scheme with a reduced value of $R_{\mathrm{g} \_ \text {main }}=11 \Omega$ was shown to have the most optimized and predictable frequency response. At $4 \mathrm{MHz}$, this control scheme achieved a harmonic content attenuation of $37 \mathrm{~dB}$ compared to an equivalent hard switching inverter. Thus, the ACPI can be employed for addressing the source of EMI in VSCs, with minimal to no impact on their efficiency.

\section{REFERENCES}

[1] B. Sarlioglu and C. T. Morris, "More Electric Aircraft: Review, Challenges, and Opportunities for Commercial Transport Aircraft," IEEE Transactions on Transportation Electrification, vol. 1, no. 1, pp. 54-64, June 2015.

[2] G. Calderon-Lopez, A. J. Forsyth, D. L. Gordon and J. R. McIntosh, "Evaluation of SiC BJTs for High-Power DC-DC Converters," IEEE Transactions on Power Electronics, vol. 29, no. 5, pp. 2474-2481, May 2014.

[3] C. R. Paul, Introduction to Electromagnetic Compatibility, 2nd ed. Hoboken, NJ, Wiley, 2006.

[4] G. Skibinski, J. Pankau, R. Sladky, and J. Campbell, "Generation, control and regulation of EMI from AC drives," IEEE Industry Applications Conference (IAS ‘97), 1997, vol. 2, pp. 1571-1583.

[5] A. Di Napoli and A. Ndokaj, "EMC and safety in vehicle drives," IEEE Power Electronics and Applications Conference (EPE 2011), 2011, pp. 1-8.

[6] J. Erdman, R. J. Kerkman, D. Schlegel and G. Skibinski, "Effect of PWM inverters on AC motor bearing currents and shaft voltages," IEEE Applied Power Electronics Conference and Exposition (APEC '95), 1995, vol.1, pp. 24-33.

[7] A. von Jouanne, P. Enjeti, and W. Gray, "The effect of long motor leads on PWM inverter fed AC motor drive systems," IEEE Applied Power Electronics Conference and Exposition (APEC '95), 1995, vol.2, pp. 592597.

[8] C. Jaeger, I. Grinbaum and J. Smajic, "Numerical simulation and measurement of common-mode and circulating bearing currents," IEEE International Conference on Electrical Machines (ICEM '16), 2016, pp. 486-491.

[9] Radio Technical Commission for Aeronautics. DO-160G: Environmental Conditions and Test Procedures for Airborne Equipment. http://www.rtca.org (2010)

[10] C. R. Paul and K. B. Hardin, "Diagnosis and reduction of conducted noise emissions," IEEE Transactions on Electromagnetic Compatibility, vol. 30, no. 4, pp. 553-560, Nov. 1988.

[11] M. Cacciato, A. Consoli, G. Scarcella, and A. Testa, "Reduction of common-mode currents in PWM inverter motor drives," IEEE Transactions on Industry Applications, vol. 35, no. 2, pp. 469-476, Mar.Apr. 1999.

[12] A. Elrayyah, K. MPK Namburi and Y. Sozer and I. Husain. "An Effective Dithering Method for Electromagnetic Interference (EMI) Reduction in Single-Phase DC/AC Inverters," IEEE Transactions on Power Electronics, vol. 29, no. 6, pp. 2798-2806, June 2014. 
[13] Y. Lobsiger and J. W. Kolar, "Closed-loop IGBT gate drive featuring highly dynamic di/dt and dv/dt control," IEEE Energy Conversion Congress and Exposition (ECCE '12), 2012, pp. 4754-4761.

[14] N. Mohan, T. Undeland, and W. Robbins, Power Electronics Converters, Applications and Design, J. Wiley \& Sons, Inc., 1995.

[15] V. Pickert, and C. M. Johnson, "Three-phase soft-switching voltage source converters for motor drives. I. Overview and analysis," IEE Proceedings on Electric Power Applications, vol. 146, no. 2, pp. 147-154, Mar. 1999.

[16] MA Ke, XU De-hong, ZHANG Tao, Seiki IGARASHI. "Divided-fixed Timing Control Method for Auxiliary Resonant Commutated Pole Softswitching Inverter," Proceedings of the CSEE, 2010, 30(18): 23-27 (in Chinese).

[17] W. Dong, J. Y. Choi, Y. Li, D. Boroyevich, F. C. Lee, J. Lai et al. "Comparative experimental evaluation of soft-switching inverter techniques for electric vehicle drive applications," IEEE Industry Applications Conference (IAS '01), 2001, vol. 3, pp. 1469-1476.

18] Huibin Zhu, Jih-Sheng Lai, A. R. Hefner, Yuqing Tang and Chingch Chen, "Modeling-based examination of conducted EMI emissions from hard and soft-switching PWM inverters," IEEE Transactions on Industry Applications, vol. 37, no. 5, pp. 1383-1393, Sep. 2001.

[19] J. Shao, R. L. Lin, F. C. Lee and D. Y. Chen, "Characterization of EMI performance for hard and soft-switched inverters," IEEE Applied Power Electronics Conference and Exposition (APEC '2000), 2000, vol. 2, pp. 1009-1014.

[20] Y. Tang, H. Zhu, B. Song, J. S. Lai and C. Chen, "EMI experimental comparison of PWM inverters between hard- and soft-switching techniques," Power Electronics in Transportation, pp. 71-77, Oct. 1998.

[21] M. R. Yazdani, H. Farzanehfard and J. Faiz, "EMI Analysis and Evaluation of an Improved ZCT Flyback Converter," IEEE Transactions on Power Electronics, vol. 26, no. 8, pp. 2326-2334, Aug. 2011.

[22] M. Mohammadi, E. Adib and M. R. Yazdani, "Family of Soft-Switching Single-Switch PWM Converters With Lossless Passive Snubber," IEEE Transactions on Industrial Electronics, vol. 62, no. 6, pp. 3473-3481, June 2015.

[23] M.E. Elbuluk and M.D. Kankam, "Potential starter/generator technologies for future aerospace applications," IEEE Aerospace and Electronic Systems Magazine, vol. 12, no. 5, pp. 24-31, May 1997.

[24] F. Costa, C. Gautier, E. Laboure, and B. Revol, Electromagnetic Compatibility in Power Electronics, ISTE Ltd and J. Wiley \& Sons, Inc., 2014.

[25] V. Pickert, and C. M. Johnson, "Three-phase soft-switching voltage source converters for motor drives. II. Fundamental limitations and critical assessment," IEE Proceedings on Electric Power Applications, vol. 146, no. 2, pp. 155-162, Mar. 1999.

[26] J. S. Lai, J. Zhang, H. Yu and H. Kouns, "Source and Load Adaptive Design for a High-Power Soft-Switching Inverter," IEEE Transactions on Power Electronics, vol. 21, no. 6, pp. 1667-1675, Nov. 2006.

[27] X. Yuan, and I. Barbi, "Analysis, designing, and experimentation of a transformer-assisted PWM zero-voltage switching pole inverter," IEEE Transactions on Power Electronics, vol. 15, no. 1, pp. 72-82, Jan 2000.

[28] I. Barbi, and D. C. Martins, "A true PWM zero-voltage switching pole, with very low additional RMS current stress," Power Electronics Specialists Conference (PESC '91), 1991, pp. 261-267.

[29] W. Yu, J. S. Lai and S. Y. Park, “An Improved Zero-Voltage Switching Inverter Using Two Coupled Magnetics in One Resonant Pole," IEEE Transactions on Power Electronics, vol. 25, no. 4, pp. 952-961, April 2010.

[30] R. W. De Doncker, and J. P. Lyons, "The auxiliary resonant commutated pole converter," IEEE Industry Applications Society Annual Meeting, 1990, vol. 2, pp. 1228-1235.

[31] W. McMurray, "Resonant snubbers with auxiliary switches," IEEE Industry Applications Society Annual Meeting, 1989, vol.1, pp. 289-834.

[32] N. Soltau, J. Lange, M. Stieneker, H. Stagge and R. W. De Doncker, "Ensuring soft-switching operation of a three-phase dual-active bridge DC-DC converter applying an auxiliary resonant-commutated pole," IEEE European Conference on Power Electronics and Applications (EPE '14), 2014, pp. 1-10.

[33] K. Ma, D. Xu, T. Zhang, and S. Igarashi, "The evaluation of control strategies for auxiliary resonant commutated pole inverter," IEEE Energy Conversion Congress and Exposition (ECCE 2009), 2009, pp. 810-816.

[34] Baoquan Kou, H. Zhang, Yinxi Jin and H. Zhang, "An improved control scheme for single-phase auxiliary resonant snubber inverter," IEEE Power Electronics and Motion Control Conference (IPEMC-ECCE Asia '16), 2016, pp. 1259-1263.

[35] Michigan State University. Electromagnetic Compatibility (EMC) Course Notes. http://www.egr.msu.edu/em/research/goali/notes/ (2007).

[36] N. Oswald, B. H. Stark, D. Holliday, C. Hargis, and B. Drury, "Analysis of shaped pulse transitions in power electronic switching waveforms for reduced EMI generation," IEEE Transactions on Industry Applications, vol. 47, no. 5, pp. 2154-2165, Sept.-Oct. 2011.

[37] A. Karvonen, T. Thiringer, P. Futane, T. Tuveson, and H. Holst "Reduction of EMI in switched mode converters by shaped pulse transitions," Society Automotive Engineers (SAE) World Congress, 2007, Paper 2007-01-0361.

[38] D. G. Holmes and Thomas A. Lipo, Pulse Width Modulation for Power Converters: Principles and Practices, J. Wiley \& Sons, Inc., 2003

[39] A. Nagel, and R. W. De Doncker, "Analytical approximations of interference spectra generated by power converters," IEEE Industry Applications Conference (IAS '97), 1997, vol. 2, pp. 1564-1570.

[40] D. M. Divan, "The resonant DC link converter-a new concept in static power conversion," IEEE Transactions on Industry Applications, vol. 25 no. 2, pp. 317-325, March 1989

[41] D. M. Divan and G. Skibinski, "Zero-switching-loss inverters for highpower applications," IEEE Transactions on Industry Applications, vol. 25, no. 4, pp. 634-643, July 1989.

[42] R. Teichmann, "Control parameter selection in auxiliary resonant commutated pole converters," IEEE Industrial Electronics Society Conference (IECON '01), 2001, vol. 2, pp. 862-869.

[43] Wei Dong, Dengming Peng, Huijie Yu, F. C. Lee and J. Lai, “A simplified control scheme for zero voltage transition (ZVT) inverter using coupled inductors," IEEE Power Electronics Specialists Conference (PESC '00), 2000, vol. 3, pp. 1221-1226.

[44] Q. Yu, and R. M. Nelms, "State plane analysis of an auxiliary resonant commutated pole inverter and implementation with load current adaptive fixed timing control," IEEE Industrial Electronics Society Conferece (IECON 02), 2002, vol.1, pp. 437-443 vol.

[45] A. Charalambous, X. Yuan, N. McNeill, Q. Yan, and N. Oswald, "EMI reduction with a soft-switched auxiliary commutated pole inverter," IEEE Energy Conversion Congress and Exposition (ECCE 2015), 2015, pp. 2650-2657.

[46] A. Charalambous, X. Yuan, N. McNeill, S. Walder, Q. Yan, and C. Frederickson, "Controlling the output voltage frequency response of the auxiliary commutated pole inverter," IEEE Industrial Electronics Society Conference (IECON 2016), 2016, pp. 3305-3310.

[47] R. Teichmann and H. Gueldner, "Analysis of transfer ratio limitations in auxiliary resonant commutated pole converters," IEEE International Power Electronics Congress (CIEP 2000), 2000, pp. 15-20.

[48] G. Rizzoli, M. Mengoni, L. Zarri, A. Tani, G. Serra and D. Casadei, "Experimental comparison of hard-switching, ZVT and SiC inverters," IEEE International Symposium on Power Electronics, Electrical Drives, Automation and Motion (SPEEDAM '16), 2016, pp. 184-191.

[49] M. Trivedi, and K. Shenai, "Internal dynamics of IGBT under zerovoltage and zero-current switching conditions," IEEE Transactions on Electron Devices, vol. 46, no. 6, pp. 1274-1282, Jun. 1999.

[50] C. Du, W. G. Hurley and D. Xu, "Design Methodology of Resonant Inductor in a ZVS Inverter," IEEE Journal of Emerging and Selected Topics in Power Electronics, vol. 3, no. 4, pp. 1142-1150, Dec. 2015.

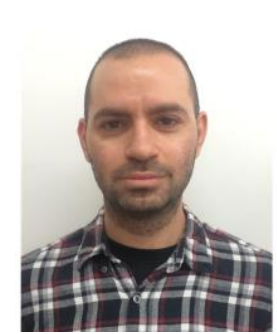

Apollo Charalambous received the Diploma (B.S and M.S.) degree in electrical engineering from the University of Patras, Patras, Greece, in 2012. He is currently working toward the Ph.D. degree in the Electrical Energy Management Group, University of Bristol, Bristol, U.K.

His research interests include power electronics, soft-switching inverters, EMI and EMC, and motor drives.

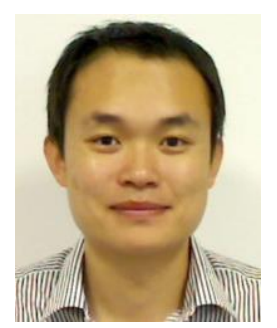

Xibo Yuan (S'09-M'11-SM'15) received the B.S degree from China University of Mining and Technology, Xuzhou, China, and the Ph.D. degree from Tsinghua University, Beijing, China, in 2005 and 2010, respectively, both in electrical engineering.

He has been a Professor since 2017 in the Electrical Energy Management Group, Department of Electrical and Electronic Engineering, University of Bristol, Bristol, U.K, where he became a Lecturer, Senior Lectuer and Reader in 2011, 2015 and 2016 respectively. He also holds a Royal Academy of Engineering/Safran Chair in Advanced Aircraft Power Generation Systems. He was a Visiting Scholar at the Center for Power Electronics Systems, Virginia Tech, Blacksburg, VA, USA, and the Institute of Energy Technology, Aalborg University, Denmark. He was 
a Postdoctoral Research Associate in the Electrical Machines and Drives Research Group, Department of Electronic and Electrical Engineering, University of Sheffield, Sheffield, U.K.

His research interests include power electronics and motor drives, wind power generation, multilevel converters, application of wide-bandgap devices, electric vehicles and more electric aircraft technologies.

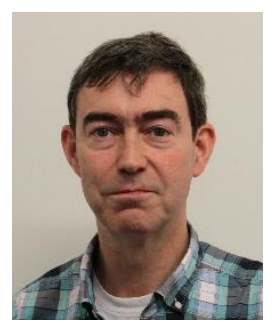

Neville McNeill received the M.Phil. and Ph.D. degrees in power electronics from Napier University, Edinburgh, Scotland, in 2003 and 2008 respectively.

His career has included periods working for electric vehicle and renewable energy companies. From 2004 to 2016 he was at the University of Bristol, Bristol, U.K., where he was latterly Senior Lecturer in Power Electronics. Since 2016 he has been with the Power Electronics, Drives and Energy Conversion (PEDEC) group in the Department of Electronic and Electrical Engineering at the University of Strathclyde, Glasgow, U.K. where he is Senior Research Fellow in Power Electronics. His main research interests are in high-efficiency multi-kilowatt power electronic conversion for aerospace, renewable energy, and electric vehicle applications.

Dr McNeill is a Member of the Institution of Engineering and Technology, a Chartered Engineer with the Engineering Council of the U.K., and a Fellow of the Higher Education Academy. 